Article

\title{
Alcohols as Substrates and Solvents for the Construction of 3-Alkoxylated-2-Oxindoles by Direct Alkoxylation of 3-Halooxindoles
}

\author{
Bing Lin ${ }^{1,+}{ }^{\dagger}$, Zhi-Yong Chen ${ }^{1,+}{ }^{,}$Huan-Huan Liu ${ }^{1}$, Qi-Di Wei ${ }^{1}$, Ting-Ting Feng ${ }^{1}$, Ying Zhou ${ }^{1, *}$, \\ Can Wang ${ }^{1}$, Xiong-Li Liu ${ }^{1, *}$ and Wei-Cheng Yuan ${ }^{2}$ \\ 1 Guizhou Medicine Edicine Edible Plant Resources Research and Development Center, College of Pharmacy, \\ Guizhou University, Guiyang 550025, China; nlin@gzu.edu.cn (B.L.); dream198896@126.com (Z.-Y.C.); \\ edithcl@sina.com (H.-H.L.); xuchwhj@163.com (Q.-D.W.); ttfeng@gzu.edu.cn (T.-T.F.); \\ ftt0809@163.com (C.W.) \\ 2 Key Laboratory for Asymmetric Synthesis \& Chirotechnology of Sichuan Province, \\ Chengdu Institute of Organic Chemistry, Chinese Academy of Sciences, Chengdu 610041, China; \\ yuanweicheng@126.com \\ * Correspondence: yingzhou71@yeah.net (Y.Z.); xlliu1@gzu.edu.cn (X.-L.L.) \\ + These two authors contributed equally to this work. \\ Academic Editor: Derek J. McPhee \\ Received: 20 April 2017; Accepted: 12 May 2017; Published: 13 May 2017
}

\begin{abstract}
Described herein is an environmentally benign method for the synthesis of multisubstituted 3-alkoxylated-2-oxindoles 3 via direct alkoxylation of 3-halooxindoles $\mathbf{1}$. A wide variety of such multisubstituted 3-alkoxylated-2-oxindole scaffolds were smoothly obtained in good yields (up to $94 \%$ ) by heating in an oil bath at $35^{\circ} \mathrm{C}$ for $24 \mathrm{~h}$. A particularly valuable feature of this method was the development of environment-friendly chemistry using alcohols 2 as both the substrates and solvents in the presence of a catalytic amount of base.
\end{abstract}

Keywords: 3-alkoxylated-2-oxindoles; 3-halooxindoles; alcohols; alkoxylation; environment-friendly chemistry

\section{Introduction}

3, $3^{\prime}$-Disubstituted oxindoles are embedded in the indole alkaloids and various clinical pharmaceuticals [1-11]. Significant efforts have been devoted by many research groups to the synthesis of 3,3'-disubstituted oxindoles. Among them, the most important and efficient approaches involve the use of electrophilic isatins/isatinimines and nucleophilic 3-monosubstituted oxindoles for the synthesis of $3,3^{\prime}$-disubstituted oxindoles (Figure 1) $[2-5,12,13]$. Despite these advances, however, the use of indol-2-ones (generated in situ from 3-halooxindoles) as electrophiles has been limited [14-28].
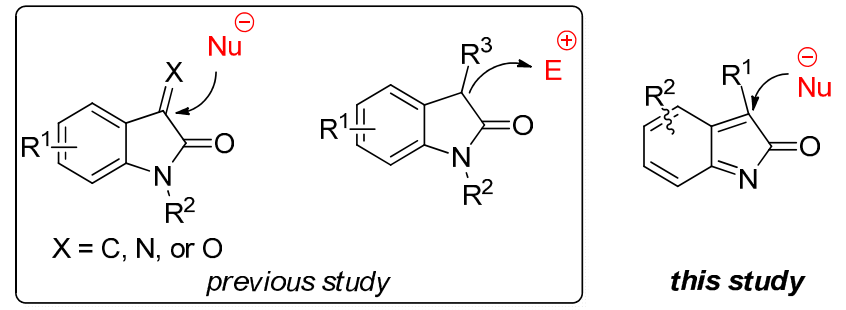

Figure 1. Strategies for the synthesis of $3,3^{\prime}$-disubstituted oxindoles. 
On the other hand, as 3,3'-disubstituted oxindole scaffolds, 3-alkoxylated-2-oxindoles possessing interesting structural properties were found in a number of biologically active synthetic and natural products (Figure 2) [29-37] Therefore, methods for alkoxylation of the oxindole nucleus are of value in medicinal chemistry and natural product synthesis. Over the past several years, although many synthetic methods have been developed for the synthesis of 3-hydroxy-2-oxindoles, existing catalytic syntheses of substituted 3-alkoxylated-2-oxindoles from simple substrates and catalysts are very few [38-41]. Worthy of note is that in 1964, Hinman and Bauman, et al. reported only a single example that described the synthesis of 3-methoxy-3-methyloxindole from 3-bromo-3-methyloxindole by treatment with 1 equivalent of $\mathrm{NaHCO}_{3}$ in $\mathrm{MeOH} /$ water (10/1). Prompted by this precedent, we have recently expanded the scope of this type of electrophile to synthesize a wide variety of 3-sulfonylated 3,3-disubstituted oxindole derivatives (Scheme 1) [21]. In this context, considering the high solubility of $\mathrm{HCl}$ in the alcoholic solvent, we supposed that a stoichiometric amount of base may be not necessary in alkoxylation of 3-halooxindoles in alcoholic solvents. We present herein the use of alcohols as both the substrates and solvents for the synthesis of 3-alkoxylated-2-oxindoles by direct alkoxylation of 3-halooxindoles using a catalytic amount of base.

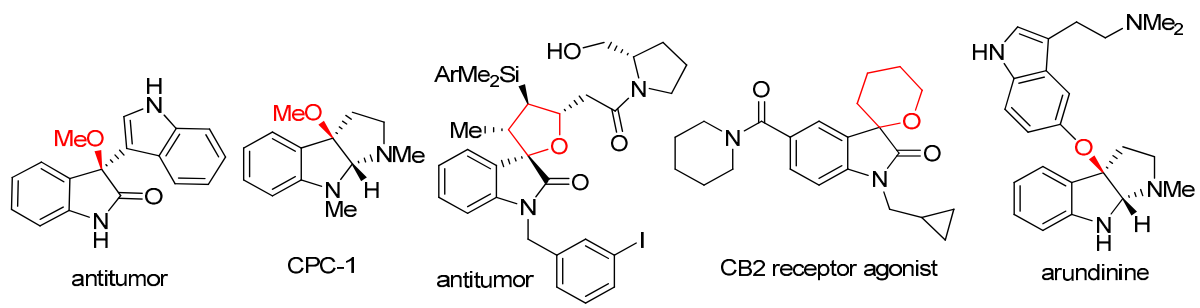

Figure 2. Representatives of the 3-alkoxylated-2-oxindolederivatives.

a) Our previous work ${ }^{24}$

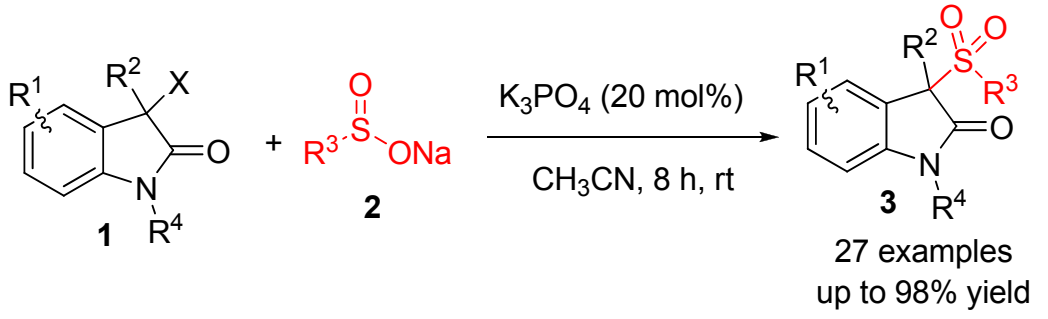

b) This work

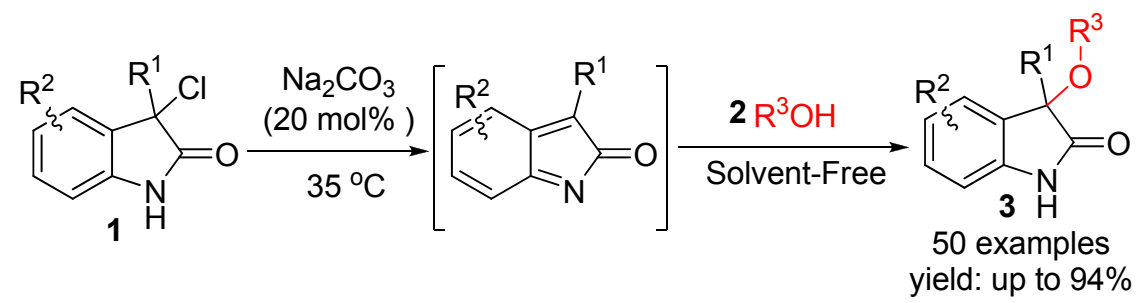

Scheme 1. Construction of 3,3'-disubstituted oxindoles using 3-halooxindoles as electrophiles.

\section{Results and Discussion}

In our initial endeavor, the 3-chloroxindole 1a was prepared via a three-step approach (Knoevenagel condensation, reduction and chlorination) using benzaldehyde and 2-oxindole as the starting materials [17]. We then investigated 3-chloroxindole 1a as a starting substrate to substantiate the feasibility of the strategy under various reaction conditions, as shown in Table 1 . The tertiary amine catalyst DABCO (entry 1, Table 1) failed to afford the desired product 3aa, providing an intractable product mixture from which no product could be identified by HRMS analysis. We then screened 
other different tertiary amines and inorganic bases (e.g., $\mathrm{Et}_{3} \mathrm{~N}, \mathrm{DBU}, \mathrm{Na}_{2} \mathrm{CO}_{3}, \mathrm{~K}_{2} \mathrm{CO}_{3}$ and $\mathrm{NaHCO}_{3}$ ) as catalysts in the reaction, and found that they can catalyse the reaction successfully leading to the desired product 3aa in moderate to good yields (entries 2-6, Table 1). In the absence of catalyst, the reaction did not well occur under otherwise identical conditions, and only starting materials remained (Table 1, entry 7). Further solvent screening demonstrated that the reaction could deliver the product 3aa preferentiallys ( $94 \%$ yield) with $\mathrm{MeOH}$ as the solvent (Table 1, entry 6). When the reaction was performed in EtOAc and THF, only 10\% and 13\% yields of 3aa were obtained, respectively (Table 1, entries 10 and 11). Considering the environmental friendliness of this chemistry and good solubility of the substrates in alcohols, we chose to use alcohols as both the substrates and solvents in the alkoxylation reactions of 3-halooxindoles.Shortening the reaction time led to the desired product 3aa in the relatively lower yields, along with some remaining starting materials (Table 1, entries 13 and 14). Further screening of the amount of the base demonstrated that the reaction could deliver the product 3 aa in $94 \%$ yield when a catalytic amount (20 mol \%) of $\mathrm{Na}_{2} \mathrm{CO}_{3}$ was employed (Table 1, entry 6 and entries 15-17). Thus, the optimal reaction conditions we established were: 3-chloroxindole 1a $(0.40 \mathrm{mmol}), 20 \mathrm{~mol} \%$ of $\mathrm{Na}_{2} \mathrm{CO}_{3}(0.08 \mathrm{mmol})$ in $4.0 \mathrm{~mL}$ of $\mathrm{MeOH} 2 \mathrm{a}$ in an oil bath at $35^{\circ} \mathrm{C} \mathrm{for} 24 \mathrm{~h}$.

Table 1. Optimization of reaction conditions ${ }^{a}$.

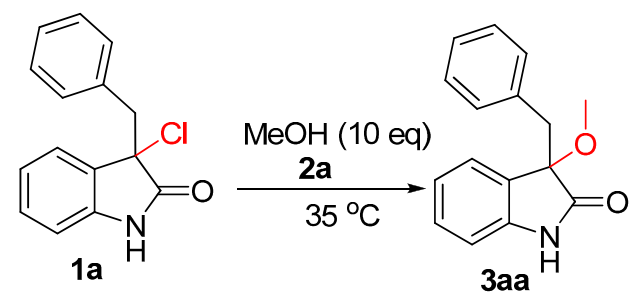

\begin{tabular}{|c|c|c|c|c|}
\hline Entry $^{a}$ & Solvent & Catalyst (20 $\mathrm{mol} \%)$ & Time (h) & Yield $^{b}(\%)$ \\
\hline $1^{c}$ & $\mathrm{MeOH}$ & $\mathrm{DABCO}$ & 24 & trace \\
\hline $2^{c}$ & $\mathrm{MeOH}$ & DBU & 24 & 54 \\
\hline $3^{c}$ & $\mathrm{MeOH}$ & $\mathrm{Et}_{3} \mathrm{~N}$ & 24 & 73 \\
\hline $4^{c}$ & $\mathrm{MeOH}$ & $\mathrm{K}_{2} \mathrm{CO}_{3}$ & 24 & 87 \\
\hline $5^{c}$ & $\mathrm{MeOH}$ & $\mathrm{NaHCO}_{3}$ & 24 & 76 \\
\hline $6^{c}$ & $\mathrm{MeOH}$ & $\mathrm{Na}_{2} \mathrm{CO}_{3}$ & 24 & 94 \\
\hline 7 & $\mathrm{MeOH}$ & None & 24 & $<10$ \\
\hline 8 & toluene & $\mathrm{Na}_{2} \mathrm{CO}_{3}$ & 24 & 61 \\
\hline 9 & DCM & $\mathrm{Na}_{2} \mathrm{CO}_{3}$ & 24 & 59 \\
\hline 10 & EtOAc & $\mathrm{Na}_{2} \mathrm{CO}_{3}$ & 24 & 10 \\
\hline 11 & THF & $\mathrm{Na}_{2} \mathrm{CO}_{3}$ & 24 & 13 \\
\hline $12^{c}$ & $\mathrm{CH}_{3} \mathrm{CN}$ & $\mathrm{Na}_{2} \mathrm{CO}_{3}$ & 24 & 51 \\
\hline $13^{c}$ & $\mathrm{MeOH}$ & $\mathrm{Na}_{2} \mathrm{CO}_{3}$ & 4 & 63 \\
\hline $14^{c}$ & $\mathrm{MeOH}$ & $\mathrm{Na}_{2} \mathrm{CO}_{3}$ & 6 & 79 \\
\hline $15^{c, d}$ & $\mathrm{MeOH}$ & $\mathrm{Na}_{2} \mathrm{CO}_{3}$ & 15 & 96 \\
\hline $16^{c, e}$ & $\mathrm{MeOH}$ & $\mathrm{Na}_{2} \mathrm{CO}_{3}$ & 48 & 78 \\
\hline $17^{c, f}$ & $\mathrm{MeOH}$ & $\mathrm{Na}_{2} \mathrm{CO}_{3}$ & 72 & 61 \\
\hline
\end{tabular}

${ }^{a}$ Unless otherwise noted, reactions were carried out with $0.4 \mathrm{mmol}$ of $\mathbf{1 a}, 2.0 \mathrm{mmol}$ of $2 \mathrm{a}, 20 \mathrm{~mol} \%$ of catalyst in the $4.0 \mathrm{~mL}$ of solvent for the specified time. ${ }^{b}$ Isolated yield after flash chromatography. ${ }^{c}$ The reaction was carried out using $\mathrm{MeOH}$ as substrate and solvent. ${ }^{d}$ The reaction was carried out using $100 \mathrm{~mol} \%$ of base $\mathrm{Na}_{2} \mathrm{CO}_{3}$. ${ }^{e}$ The reaction was carried out using $10 \mathrm{~mol} \%$ of base $\mathrm{Na}_{2} \mathrm{CO}_{3} .{ }^{f}$ The reaction was carried out using $5 \mathrm{~mol} \%$ of base $\mathrm{Na}_{2} \mathrm{CO}_{3}$.

With the best reaction conditions in hands, we next turned our interest to the reaction scope, and the results are summarized in Table 2. $\mathrm{MeOH}$ (2a) was first used as a standard substrate to probe the reactivity of different 3-chloroxindoles 1 in this reaction. Significant structural variation in the oxindole system could be accommodated in this reaction. For example, electron-rich (Table 2, 3ba and 3ca) and electron-poor (Table 2, 3da-3ja) substituents incorporated on the phenyl group or the benzo moiety of the oxindole core were perfectly tolerated under the conditions. 
Table 2. Synthesis of 3-alkoxylated-2-oxindoles $3^{a, b}$.

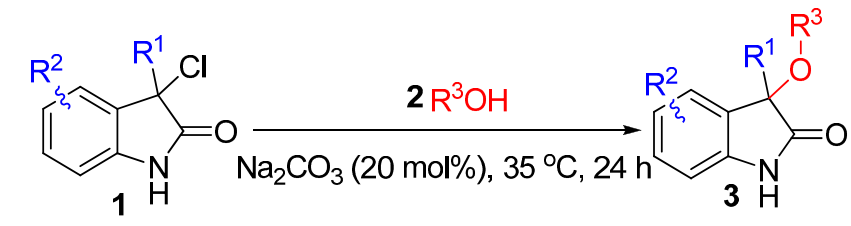

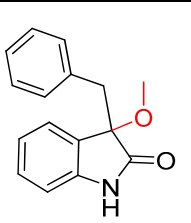

3aa, yield: $94 \%$<smiles>COC1(Cc2ccccc2Cl)C(=O)Nc2ccccc21</smiles>

3fa, yield: $93 \%$<smiles>CCOC1(Cc2ccccc2)C(=O)Nc2ccccc21</smiles>

3ab, yield: $89 \%$

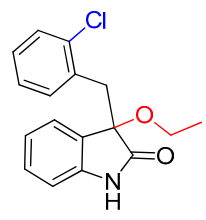

3fb, yield: $90 \%$

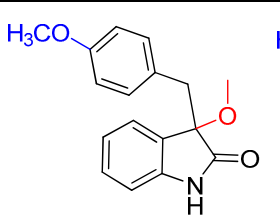

3ba, yield: $92 \%$<smiles>COCC1(Cc2ccc(C)cc2)C(=O)Nc2ccccc21</smiles>

3ca, yield: $92 \%$<smiles>COC1(Cc2cccc(F)c2)C(=O)Nc2ccccc21</smiles>

3ha, yield: 90\%

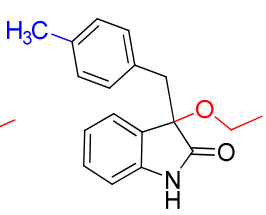

3cb, yield: $91 \%$

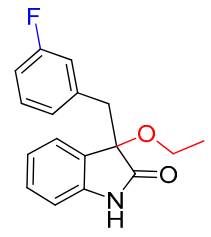

3hb, yield: $89 \%$<smiles>CNc1ccccc1C(COC)(Cc1ccc(Br)cc1)OC</smiles>

3da, yield: $91 \%$

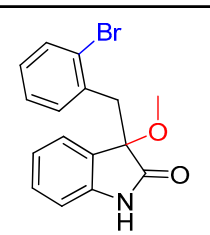

3ea, yield: 91\%<smiles>CCC(COC)(Cc1ccccc1)c1cccc(Cl)c1</smiles>

3ia, yield: $90 \%$

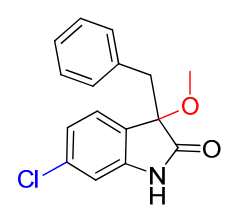

3ja, yield: $91 \%$

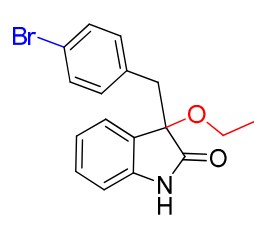

3db, yield: $90 \%$

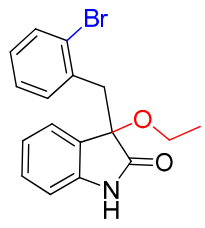

3eb, yield: $88 \%$

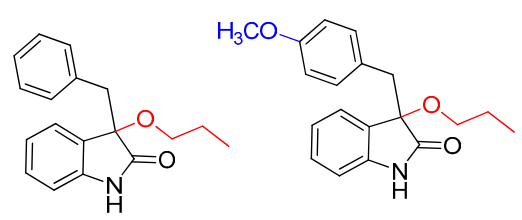

3ac, yield: $86 \%$ 3bc, yield: $82 \%$

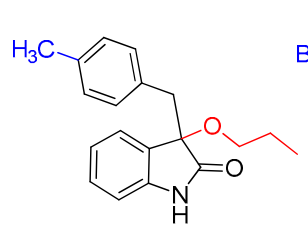

3cc, yield: $83 \%$

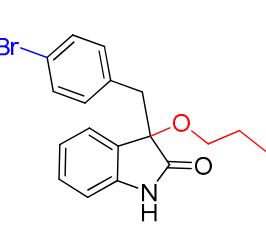

3dc, yield: $82 \%$

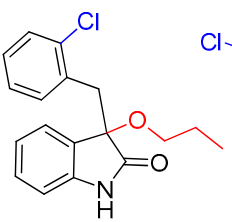

3fc, yield: $80 \%$

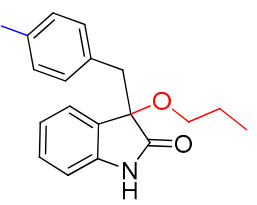

3gc, yield: $84 \%$<smiles>CC(C)OC1(Cc2ccc(Br)cc2)C(=O)Nc2ccccc21</smiles>

3dd, yield: $77 \%$

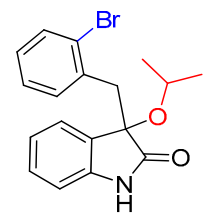

3ed, yield: $74 \%$

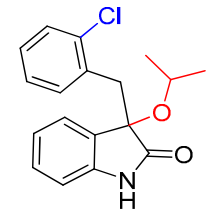

3fd, yield: $70 \%$<smiles>CC(C)OC1(Cc2ccc(Cl)cc2)C(=O)Nc2ccccc21</smiles>

3gd, yield: $72 \%$

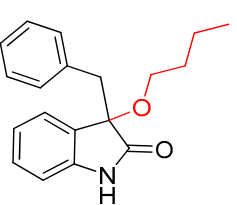

3ae, yield: $80 \%$

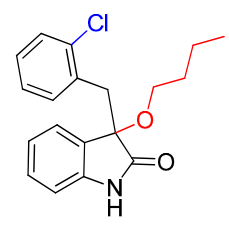

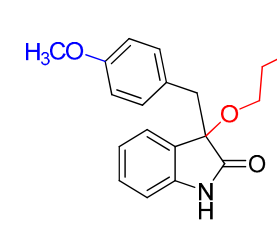

3be, yield: $83 \%$

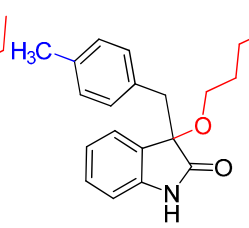

3ce, yield: $85 \%$

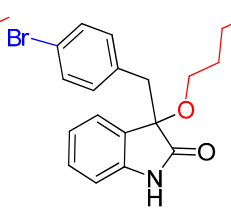

3de, yield: $84 \%$

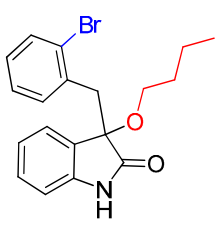

3ee, yield: $80 \%$

3fe, yield: $81 \%$ 
Table 2. Cont<smiles>CCCCOC1(Cc2ccc(Cl)cc2)C(=O)Nc2ccccc21</smiles>

3ge, yield: $83 \%$

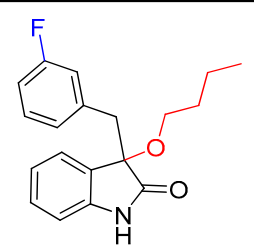

3he, yield: $74 \%$<smiles>O=C1Nc2ccccc2C1(Cc1ccccc1)Cc1ccccc1</smiles>

3af, yield: $84 \%$<smiles>COc1ccc(CC2(Cc3ccccc3)C(=O)Nc3ccccc32)cc1</smiles>

3bf, yield: $85 \%$<smiles>Cc1ccc(CC2(Cc3ccccc3)C(=O)Nc3ccccc32)cc1</smiles>

3cf, yield: $80 \%$<smiles>O=C1Nc2ccccc2C1(Cc1ccccc1)OCc1ccccc1</smiles>

3hf, yield: $75 \%$<smiles>O=C1Nc2ccccc2C1(Cc1ccccc1)Cc1ccc(Br)cc1</smiles>

3df, yield: $82 \%$<smiles>O=C1Nc2ccccc2C1(Cc1ccccc1)OCc1ccccc1</smiles>

3ef, yield: $81 \%$
43<smiles>O=C1Nc2ccccc2C1(Cc1ccccc1)OCc1ccccc1</smiles><smiles>O=C1Nc2ccccc2C1(Cc1ccc(Cl)cc1)OCc1ccccc1</smiles>

3gf, yield: $83 \%$<smiles>O=C1Nc2ccccc2C1(Cc1ccccc1)OCC(O)CO</smiles>

3if, yield: $82 \%$

3jf, yield: $85 \%$<smiles>OC(Cl)(Cl)Cl</smiles><smiles>O=C1Nc2ccccc2C1(Cc1ccccc1)OCCO</smiles>

3gg, yield: $87 \%$

3ah, yield: $51 \%^{c}$, dr: $1: 1$

${ }^{a}$ Unless otherwise noted, reactions were carried out with $0.4 \mathrm{mmol}$ of 1 and $20 \mathrm{~mol} \%$ of catalyst $\mathrm{Na}_{2} \mathrm{CO}_{3}$ in the $4.0 \mathrm{~mL}$ of alcohol 2 in oil bath at $35^{\circ} \mathrm{C}$ for $24 \mathrm{~h} .{ }^{b}$ Isolated yield after flash chromatography. ${ }^{c}$ This reaction was carried out in oil bath at $45^{\circ} \mathrm{C}$ for $24 \mathrm{~h}$.

The generality of the reaction was further demonstrated by using a variety of alcohols 2 , clearly indicating that all of the reactions proceeded smoothly under the optimal conditions, producing the desired products 3 in moderate to good yields (Table 2, 3ab-3ah), regardless of the electronic nature of the chloroxindoles $\mathbf{1}$. It is noteworthy that the bulky isopropyl alcohol (2d) led to deleterious effects on the reactivity, affording the desired products in moderate yields (Table 2, 3dd-3gd). In addition, our attempts to identify the alkoxylation reactions of 3-aryl or 3-arylmethyl substituted $\mathrm{N}$-Boc-oxindoles 1 using $\mathrm{MeOH}$ as substrate and solvent were in vain (Scheme 2). Furthermore, using 3-aryl substituted $\mathrm{N}$-Me-oxindole $1 \mathrm{~m}$ as substrate it proved difficult to obtain the pure compound $1 \mathrm{ma}$, and we always obtained an intractable product mixture (Scheme 2).
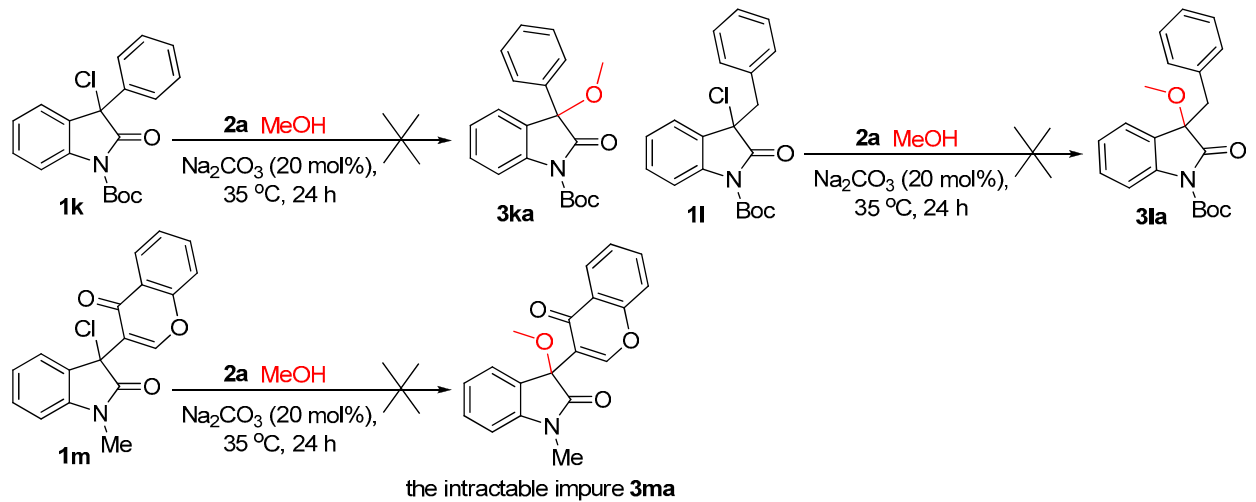

Scheme 2. Contrast experiments with different types of substrate oxindoles $\mathbf{1}$ using $\mathrm{MeOH}$ as substrate and solvent. 
In order to further explore the scope of the substrates, we also chose bromooxindole $\mathbf{1}^{\prime} \mathbf{a}$ as a test substrate for this transformation. To our delight, the reaction proceeded well to give the desired product 3aa in good yield (88\%) under the standard reaction conditions (Scheme 3).

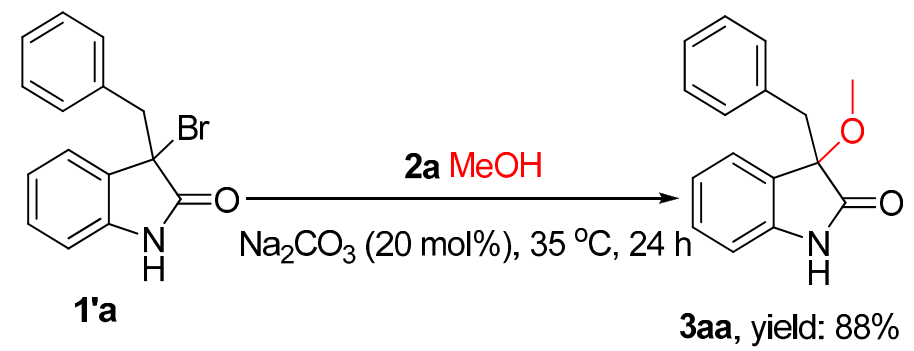

Scheme 3. Bromooxindole $\mathbf{1}^{\prime} \mathbf{a}$ as a test substrate for this transformation.

The significance and the high efficiency of the current protocol were demonstrated by a gram-scale synthesis of 3aa. The alkoxylation of 3-chloroxindole 1a proceeded cleanly on a $4.0 \mathrm{mmol}$ scale (1.03 $\mathrm{g}$ of 1a) in oil bath at $35^{\circ} \mathrm{C}$ for $48 \mathrm{~h}$. As outlined in Scheme 4 , the corresponding adduct 3aa was obtained smoothly in $92 \%$ yield, which was similar to those observed in a previous investigation (entry 1 of Table 2).

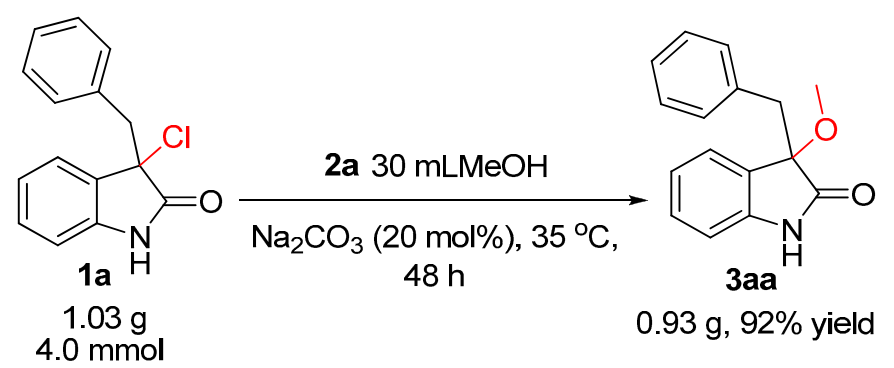

Scheme 4. The alkoxylation of chloroxindole 1a on a gram scale.

\section{Experimental Section}

\subsection{General}

The ${ }^{1} \mathrm{H}$ and ${ }^{13} \mathrm{C}$ NMR spectra were recorded on Bruker Avance DMX $400 \mathrm{MHz}$ or $500 \mathrm{M}$ NMR spectrometers (Bruker, Billerica, MA, USA) in $\mathrm{CDCl}_{3}$ using TMS as internal standard. Chemical shifts were reported as $\delta$ values (ppm). High-resolution mass spectra (HRMS-ESI) were obtained on a Micro ${ }^{\mathrm{TM}}$ Q-TOF Mass Spectrometer (Waters, Milford, MA, USA). Melting points were uncorrected and recorded on an Electothermal 9100 digital melting point apparatus (Electothermal, Stone, UK). Reagents were purchased from commercial sources and were used as received unless mentioned otherwise. Reactions were monitored by thin layer chromatography using silica gel $\mathrm{GF}_{254}$ plates. Column chromatography was performed on silica gel (300-400 mesh).

\subsection{General Experimental Procedures for Synthesis of 3-Alkoxylated-2-Oxindoles 3}

In an ordinary vial equipped with a magnetic stirring bar was added 3-chloroxindole $\mathbf{1}(0.4 \mathrm{mmol})$, $20 \mathrm{~mol} \%$ of catalyst $\mathrm{Na}_{2} \mathrm{CO}_{3}(8.5 \mathrm{mg}, 0.08 \mathrm{mmol})$ and $4.0 \mathrm{~mL}$ of alcohol 2 . The reaction mixture was stirred in oil bath at $35^{\circ} \mathrm{C}$ for $24 \mathrm{~h}$. After completion of the reaction, as indicated by TLC, the removal of solvent and purification by flash column chromatography (hexane/EtOAc $=10: 1 \sim 6: 1$ ) was carried out to furnish the corresponding products 3. 


\subsection{Characterization Data of Compounds 3}<smiles>COC1(Cc2ccccc2)C(=O)Nc2ccccc21</smiles>

3-Benzyl-3-methoxyindolin-2-one (3aa). Light orange solid, m.p. $120.3-122.5{ }^{\circ} \mathrm{C}$; yield 94\%; ${ }^{1} \mathrm{H}-\mathrm{NMR}$ $\left(\mathrm{CDCl}_{3}\right) \delta: 3.08(\mathrm{~s}, 3 \mathrm{H}), 3.11(\mathrm{~d}, J=12.8 \mathrm{~Hz}, 1 \mathrm{H}), 3.32(\mathrm{~d}, J=12.8 \mathrm{~Hz}, 1 \mathrm{H}), 6.80-6.83(\mathrm{~m}, 1 \mathrm{H}), 6.93-6.96$ $(\mathrm{m}, 2 \mathrm{H}), 7.02-7.11(\mathrm{~m}, 5 \mathrm{H}), 7.22-7.26(\mathrm{~m}, 1 \mathrm{H}), 9.13$ (br s, $1 \mathrm{H}) ;{ }^{13} \mathrm{C}-\mathrm{NMR}\left(\mathrm{CDCl}_{3}\right)$ 8: 43.6, 5.3.3, 84.2, 110.4, 122.6, 125.3, 126.3, 126.7, 127.6, 129.8, 130.6, 133.9, 141.2, 178.6; HRMS (ESI-TOF) $m / z$ : Calcd. for $\mathrm{C}_{16} \mathrm{H}_{15} \mathrm{NNaO}_{2}[\mathrm{M}+\mathrm{Na}]^{+}:$276.1000; Found: 276.1004. Spectra are in Supplementary Materials.<smiles>COc1ccc(CC2(OC)C(=O)Nc3ccccc32)cc1</smiles>

3-Methoxy-3-(4-methoxybenzyl)indolin-2-one (3ba). Light orange solid, m.p. 142.3-144.1 ${ }^{\circ} \mathrm{C}$; yield 92\%; ${ }^{1} \mathrm{H}-\mathrm{NMR}\left(\mathrm{CDCl}_{3}\right) \delta: 3.03(\mathrm{~d}, J=12.8 \mathrm{~Hz}, 1 \mathrm{H}), 3.05(\mathrm{~s}, 3 \mathrm{H}), 3.23(\mathrm{~d}, J=12.8 \mathrm{~Hz}, 1 \mathrm{H}), 3.66(\mathrm{~s}, 3 \mathrm{H}), 6.57-6.61$ $(\mathrm{m}, 2 \mathrm{H}), 6.79-6.85(\mathrm{~m}, 3 \mathrm{H}), 7.01-7.03(\mathrm{~m}, 2 \mathrm{H}), 7.20-7.25(\mathrm{~m}, 1 \mathrm{H}), 8.98($ br s, $1 \mathrm{H}) ;{ }^{13} \mathrm{C}-\mathrm{NMR}\left(\mathrm{CDCl}_{3}\right)$ $\delta: 42.9,53.4,55.1,84.4,110.5,113.1,122.7,125.4,126.0,126.6,129.9,131.7,141.4,158.5,178.8$; HRMS (ESI-TOF) $m / z$ : Calcd. for $\mathrm{C}_{17} \mathrm{H}_{17} \mathrm{NNaO}_{3}[\mathrm{M}+\mathrm{Na}]^{+}:$306.1106; Found: 306.1107.<smiles>COC1(Cc2ccc(C)cc2)C(=O)Nc2ccccc21</smiles>

3-Methoxy-3-(4-methylbenzyl)indolin-2-one (3ca). Light orange solid, m.p. 130.7-134.2 ${ }^{\circ}$; yield 91\%; ${ }^{1} \mathrm{H}-\mathrm{NMR}\left(\mathrm{CDCl}_{3}\right) \delta: 2.13(\mathrm{~s}, 3 \mathrm{H}), 2.97-3.01(\mathrm{~m}, 4 \mathrm{H}), 3.19(\mathrm{~d}, J=12.8 \mathrm{~Hz}, 1 \mathrm{H}), 6.72-6.81(\mathrm{~m}, 5 \mathrm{H}), 6.94-6.99$

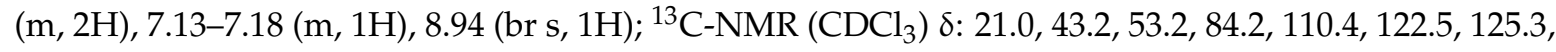
126.5, 128.3, 129.7, 130.4, 130.7, 136.2, 141.2, 178.7; HRMS (ESI-TOF) $m / z$ : Calcd. for $\mathrm{C}_{17} \mathrm{H}_{17} \mathrm{NNaO}_{2}$ $[\mathrm{M}+\mathrm{Na}]^{+}:$290.1157; Found: 290.1154 .<smiles>COC1(Cc2ccc(Br)cc2)C(=O)Nc2ccccc21</smiles>

3-(4-Bromobenzyl)-3-methoxyindolin-2-one (3da). Light orange solid, m.p. 120.3-122.4 ${ }^{\circ}$; yield 91\%; ${ }^{1} \mathrm{H}-\mathrm{NMR}\left(\mathrm{CDCl}_{3}\right) \delta: 3.05(\mathrm{~d}, J=13.2 \mathrm{~Hz}, 1 \mathrm{H}), 3.08(\mathrm{~s}, 3 \mathrm{H}), 3.26(\mathrm{~d}, J=13.2 \mathrm{~Hz}, 1 \mathrm{H}), 6.81-6.84(\mathrm{~m}$, 3H), 7.03-7.06 (m, 2H), 7.20-7.26 (m, 3H), 8.82 (br s, $1 \mathrm{H}) ;{ }^{13} \mathrm{C}-\mathrm{NMR}\left(\mathrm{CDCl}_{3}\right)$ 8: 43.0, 53.3, 83.8, 110.5, 121.0, 122.7, 125.2, 126.0, 130.0, 130.8, 132.3, 132.9, 141.0, 178.2; HRMS (ESI-TOF) $m / z$ : Calcd. for $\mathrm{C}_{16} \mathrm{H}_{14} \mathrm{BrNNaO}_{2}[\mathrm{M}+\mathrm{Na}]^{+}$: 354.0106; Found: 354.0106. 
<smiles>COC1(Cc2ccccc2Br)C(=O)Nc2ccccc21</smiles>

3-(2-Bromobenzyl)-3-methoxyindolin-2-one (3ea). Light orange solid, m.p. 184.5-187.1 ${ }^{\circ}$; yield 91\%; ${ }^{1} \mathrm{H}-\mathrm{NMR}\left(\mathrm{CDCl}_{3}\right) \delta: 3.09(\mathrm{~s}, 3 \mathrm{H}), 3.36(\mathrm{~d}, J=11.2 \mathrm{~Hz}, 1 \mathrm{H}), 3.48(\mathrm{~d}, J=11.2 \mathrm{~Hz}, 1 \mathrm{H}), 6.68(\mathrm{~d}, J=6.0 \mathrm{~Hz}$, 1H), 6.89-6.95 (m, 2H), 7.05-7.09 (m, 1H), 7.20-7.27 (m, 2H), 7.40-7.42 (m, 1H), 7.48-7.50 (m, 1H), 9.32 (br s, $1 \mathrm{H}) ;{ }^{13} \mathrm{C}-\mathrm{NMR}\left(\mathrm{CDCl}_{3}\right) \delta: 42.0,53.2,83.2,110.5,122.6,125.6,126.8,128.5,129.8,132.4,132.8,134.4$, 140.9, 179.0; HRMS (ESI-TOF) $m / z$ : Calcd. for $\mathrm{C}_{16} \mathrm{H}_{14} \mathrm{BrNNaO}_{2}[\mathrm{M}+\mathrm{Na}]^{+}$: 354.0106; Found: 354.0105.<smiles>COC1(Cc2ccccc2Cl)C(=O)Nc2ccccc21</smiles>

3-(2-Chlorobenzyl)-3-methoxyindolin-2-one (3fa). Light orange solid, m.p. $138.2-139.8{ }^{\circ} \mathrm{C}$; yield 93\%; ${ }^{1} \mathrm{H}-\mathrm{NMR}\left(\mathrm{CDCl}_{3}\right) \delta: 3.09(\mathrm{~s}, 3 \mathrm{H}), 3.35(\mathrm{~d}, J=13.5 \mathrm{~Hz}, 1 \mathrm{H}), 3.47(\mathrm{~d}, J=13.5 \mathrm{~Hz}, 1 \mathrm{H}), 6.77(\mathrm{~d}, J=7.2 \mathrm{~Hz}$, 1H), 6.89-6.97 (m, 2H), 7.13-7.26 (m, 4H), 7.42-7.44 (m, 1H), 9.40 (br s, 1H); ${ }^{13} \mathrm{C}-\mathrm{NMR}\left(\mathrm{CDCl}_{3}\right) \delta: 39.4$, 53.2, 83.3, 110.4, 122.6, 125.5, 125.9, 126.2, 128.3, 129.1, 129.8, 132.5, 132.7, 135.3, 140.5, 141.0, 179.0; HRMS (ESI-TOF) $m / z$ : Calcd. for $\mathrm{C}_{16} \mathrm{H}_{14} \mathrm{ClNNaO}_{2}[\mathrm{M}+\mathrm{Na}]^{+}: 310.0611$; Found: 310.0614 .<smiles>COC1(Cc2ccc(Cl)cc2)C(=O)Nc2ccccc21</smiles>

3-(4-Chlorobenzyl)-3-methoxyindolin-2-one (3ga). Light orange solid, m.p. 125.2-127.3 ${ }^{\circ} \mathrm{C}$; yield 91\%; ${ }^{1} \mathrm{H}-\mathrm{NMR}\left(\mathrm{CDCl}_{3}\right) \delta: 3.05(\mathrm{~d}, J=12.8 \mathrm{~Hz}, 1 \mathrm{H}), 3.08(\mathrm{~s}, 3 \mathrm{H}), 3.27(\mathrm{~d}, J=12.8 \mathrm{~Hz}, 1 \mathrm{H}), 6.83(\mathrm{~d}, J=7.6 \mathrm{~Hz}$, $1 \mathrm{H}), 6.88(\mathrm{~d}, J=8.4 \mathrm{~Hz}, 2 \mathrm{H}), 7.02-7.08(\mathrm{~m}, 4 \mathrm{H}), 7.24-7.28(\mathrm{~m}, 1 \mathrm{H}), 8.93(\mathrm{br} \mathrm{s}, 1 \mathrm{H}) ;{ }^{13} \mathrm{C}-\mathrm{NMR}\left(\mathrm{CDCl}_{3}\right) \delta$ : $42.9,53.3,83.9,110.5,122.8,125.3,126.1,127.8,130.0,131.9,132.4,132.8,141.1,178.3$; HRMS (ESI-TOF) $m / z$ : Calcd. for $\mathrm{C}_{16} \mathrm{H}_{14} \mathrm{ClNNaO}_{2}[\mathrm{M}+\mathrm{Na}]^{+}: 310.0611$; Found: 310.0610 .<smiles>COC1(Cc2cccc(F)c2)C(=O)Nc2ccccc21</smiles>

3-(3-Fluorobenzyl)-3-methoxyindolin-2-one (3ha). Light orange solid, m.p. 135.7-138.2 ${ }^{\circ}$; yield 90\%; ${ }^{1} \mathrm{H}-\mathrm{NMR}\left(\mathrm{CDCl}_{3}, 500 \mathrm{MHz}\right) \delta: 3.07(\mathrm{~d}, J=13.5 \mathrm{~Hz}, 1 \mathrm{H}), 3.09(\mathrm{~s}, 3 \mathrm{H}), 3.31(\mathrm{~d}, J=13.5 \mathrm{~Hz}, 1 \mathrm{H}), 6.70-6.75$ (m, 2H), 6.83-6.85 (m, 2H), 6.98-7.06 (m, 3H), 7.24-7.27 (m, 1H), 9.12 (br s, 1H); ${ }^{13} \mathrm{C}-\mathrm{NMR}\left(\mathrm{CDCl}_{3}\right.$, $125 \mathrm{MHz}) \delta: 43.2,53.3,83.8,110.5,110.6,113.7\left(\mathrm{~d}, J_{C F}=20.8 \mathrm{~Hz}\right), 117.4\left(\mathrm{~d}, J_{C F}=21.3 \mathrm{~Hz}\right), 122.7,125.3$, 126.0, 126.3, 126.4, 128.9, 129.0, 130.0, 136.5, 136.6, 141.1, 141.2, 162.1 (d, $\left.J_{C F}=245.8 \mathrm{~Hz}\right), 178.5$; HRMS (ESI-TOF) $m / z$ : Calcd. for $\mathrm{C}_{16} \mathrm{H}_{14} \mathrm{FNNaO}_{2}[\mathrm{M}+\mathrm{Na}]^{+}:$294.0906; Found: 294.0908 . 
<smiles>COC1(Cc2ccccc2)C(=O)Nc2ccc(Cl)cc21</smiles>

3-Benzyl-5-chloro-3-methoxyindolin-2-one (3ia). Light orange solid, m.p. 130.4-133.3 ${ }^{\circ} \mathrm{C}$; yield 90\%; ${ }^{1} \mathrm{H}-\mathrm{NMR}\left(\mathrm{CDCl}_{3}\right)$ 8: $3.00(\mathrm{~s}, 3 \mathrm{H}), 3.03(\mathrm{~d}, J=12.8 \mathrm{~Hz}, 1 \mathrm{H}), 3.21(\mathrm{~d}, J=12.8 \mathrm{~Hz}, 1 \mathrm{H}), 6.66(\mathrm{~d}, J=8.4 \mathrm{~Hz}$, 1H), 6.87-6.92 (m, 3H), 7.01-7.08 (m, 3H), 7.13-7.19 (m, 1H), $8.83(\mathrm{br} \mathrm{s}, 1 \mathrm{H}) ;{ }^{13} \mathrm{C}-\mathrm{NMR}\left(\mathrm{CDCl}_{3}\right)$ 8: 43.6, 53.5, 84.3, 111.4, 125.6, 127.0, 127.8, 128.2, 128.3, 129.8, 130.5, 133.4, 139.6, 178.2; HRMS (ESI-TOF) $m / z$ : Calcd. for $\mathrm{C}_{16} \mathrm{H}_{14} \mathrm{ClNNaO}_{2}[\mathrm{M}+\mathrm{Na}]^{+}$: 310.0611; Found: 310.0611 .<smiles>COC1(Cc2ccccc2)C(=O)Nc2cc(Cl)ccc21</smiles>

3-Benzyl-6-chloro-3-methoxyindolin-2-one (3ja). Light orange solid, m.p. $198.4-201.9{ }^{\circ} \mathrm{C}$; yield 91\%; ${ }^{1} \mathrm{H}-\mathrm{NMR}\left(\mathrm{DMSO}-d_{6}\right) \delta: 2.91(\mathrm{~s}, 3 \mathrm{H}), 3.00(\mathrm{~d}, J=12.8 \mathrm{~Hz}, 1 \mathrm{H}), 3.20(\mathrm{~d}, J=12.8 \mathrm{~Hz}, 1 \mathrm{H}), 6.66(\mathrm{~s}, 1 \mathrm{H})$, 6.67-6.90 (m, 2H), $7.03(\mathrm{~d}, J=2.0 \mathrm{~Hz}, 1 \mathrm{H}), 7.09-7.13(\mathrm{~m}, 4 \mathrm{H}), 10.5(\mathrm{br} \mathrm{s}, 1 \mathrm{H}) ;{ }^{13} \mathrm{C}-\mathrm{NMR}$ (DMSO- $\left.d_{6}\right) \delta$ : 42.3, 52.3, 83.1, 109.9, 121.5, 125.0, 126.6, 126.8, 127.7, 130.3, 134.0, 134.1, 143.9, 176.2; HRMS (ESI-TOF) $m / z$ : Calcd. for $\mathrm{C}_{16} \mathrm{H}_{14} \mathrm{ClNNaO}_{2}[\mathrm{M}+\mathrm{Na}]^{+}$: 310.0611; Found: 310.0611 .<smiles>CCOC1(Cc2ccccc2)C(=O)Nc2ccccc21</smiles>

3-Benzyl-3-ethoxyindolin-2-one (3ab). Light orange solid, m.p. $112.0-113.8{ }^{\circ} \mathrm{C}$; yield $89 \% ;{ }^{1} \mathrm{H}-\mathrm{NMR}$ $\left(\mathrm{CDCl}_{3}\right)$ 8: 1.14-1.18 (m, 3H), 3.10-3.15 (m, 2H), 3.22-3.26 (m, 1H), $3.31(\mathrm{~d}, J=12.8 \mathrm{~Hz}, 1 \mathrm{H}), 6.78$ $(\mathrm{d}, J=7.6 \mathrm{~Hz}, 1 \mathrm{H}), 6.92-6.93(\mathrm{~m}, 2 \mathrm{H}), 6.94-7.10(\mathrm{~m}, 5 \mathrm{H}), 7.20-7.26(\mathrm{~m}, 1 \mathrm{H}), 9.01$ (br s, $1 \mathrm{H}) ;{ }^{13} \mathrm{C}-\mathrm{NMR}$ $\left(\mathrm{CDCl}_{3}\right)$ 8: 15.3, 43.8, 61.2, 83.6, 110.3, 122.5, 125.1, 126.7, 127.1, 127.6, 129.6, 130.5, 134.0, 141.0, 179.0; HRMS (ESI-TOF) $m / z$ : Calcd. for $\mathrm{C}_{17} \mathrm{H}_{17} \mathrm{NNaO}_{2}[\mathrm{M}+\mathrm{Na}]^{+}: 290.1157$; Found: 290.1154 .<smiles>COc1ccc(CC2(OC)C(=O)Nc3ccccc32)cc1</smiles>

3-Ethoxy-3-(4-methoxybenzyl)indolin-2-one (3bb). Light orange solid, m.p. 151.2-153.1 ${ }^{\circ} \mathrm{C}$; yield 92\%; ${ }^{1} \mathrm{H}-\mathrm{NMR}\left(\mathrm{CDCl}_{3}\right)$ 8: 1.12-1.16 (m, 3H), 3.02-3.12 (m, 2H), 3.19-3.26 (m, 2H), $3.65(\mathrm{~s}, 3 \mathrm{H}), 6.56-6.58$ $(\mathrm{m}, 2 \mathrm{H}), 6.76-6.84(\mathrm{~m}, 3 \mathrm{H}), 7.01-7.06(\mathrm{~m}, 2 \mathrm{H}), 7.18-7.25(\mathrm{~m}, 1 \mathrm{H}), 8.93(\mathrm{br} \mathrm{s}, 1 \mathrm{H}) ;{ }^{13} \mathrm{C}-\mathrm{NMR}\left(\mathrm{CDCl}_{3}\right) \delta$ : 15.5, 43.1, 55.1, 61.3, 83.8, 110.4, 113.1, 122.6, 125.2, 126.1, 127.5, 129.7, 131.6, 141.2, 158.4, 179.1; HRMS (ESI-TOF) $m / z$ : Calcd. for $\mathrm{C}_{18} \mathrm{H}_{19} \mathrm{NNaO}_{3}[\mathrm{M}+\mathrm{Na}]^{+}$: 320.1263; Found: 320.1263 . 


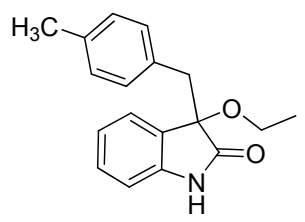

3-Ethoxy-3-(4-methylbenzyl)indolin-2-one (3cb). Light orange solid, m.p. $138.8-141.9{ }^{\circ} \mathrm{C}$; yield 91\%; ${ }^{1} \mathrm{H}-\mathrm{NMR}\left(\mathrm{CDCl}_{3}\right)$ 8: 1.07-1.10 (m, 3H), $2.13(\mathrm{~s}, 3 \mathrm{H}), 2.99-3.07(\mathrm{~m}, 2 \mathrm{H}), 3.14-3.22(\mathrm{~m}, 2 \mathrm{H}), 6.69-6.79(\mathrm{~m}$, 5H), 6.94-7.01 (m, 2H), 7.12-7.18 (m, 1H), $8.82(\mathrm{br} \mathrm{s}, 1 \mathrm{H}) ;{ }^{13} \mathrm{C}-\mathrm{NMR}\left(\mathrm{CDCl}_{3}\right)$ 8: 15.3, 21.0, 43.4, 61.1, 83.6, 110.2, 122.5, 125.1, 127.3, 128.3, 129.6, 130.4, 130.8, 136.1, 141.0, 178.9; HRMS (ESI-TOF) $m / z$ : Calcd. for $\mathrm{C}_{18} \mathrm{H}_{19} \mathrm{NNaO}_{2}[\mathrm{M}+\mathrm{Na}]^{+}$: 304.1313; Found: 304.1315 .

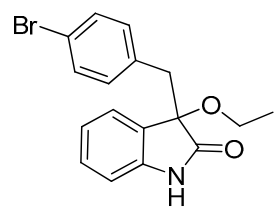

3-(4-Bromobenzyl)-3-ethoxyindolin-2-one (3db). Light orange solid, m.p. $142.1-144.6{ }^{\circ} \mathrm{C}$; yield 90\%; ${ }^{1} \mathrm{H}-\mathrm{NMR}\left(\mathrm{CDCl}_{3}\right) \delta: 1.15-1.18(\mathrm{~m}, 3 \mathrm{H}), 3.04(\mathrm{~d}, J=13.2 \mathrm{~Hz}, 1 \mathrm{H}), 3.11-3.15(\mathrm{~m}, 1 \mathrm{H}), 3.22-3.28(\mathrm{~m}, 2 \mathrm{H})$, 6.78-6.84 (m, 3H), 7.03-7.05 (m, 2H), 7.18-7.27 (m, 3H), 8.82 (br s, 1H); ${ }^{13} \mathrm{C}-\mathrm{NMR}\left(\mathrm{CDCl}_{3}\right)$ ): 15.3, 43.2, 61.3, 83.2, 110.4, 120.9, 122.7, 125.1, 126.8, 129.8, 130.7, 132.3, 133.0, 140.8, 178.6; HRMS (ESI-TOF) $m / z$ : Calcd. for $\mathrm{C}_{17} \mathrm{H}_{16} \mathrm{BrNNaO}_{2}[\mathrm{M}+\mathrm{Na}]^{+}$: 368.0262; Found: 368.0265 .

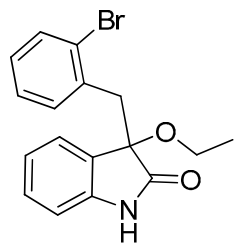

3-(2-Bromobenzyl)-3-ethoxyindolin-2-one (3eb). Light orange solid, m.p. $185.1-186.2{ }^{\circ} \mathrm{C}$; yield 88\%; ${ }^{1} \mathrm{H}-\mathrm{NMR}\left(\mathrm{CDCl}_{3}\right)$ 8: 1.14-1.18 (m, 3H), 3.07-3.11 (m, $\left.1 \mathrm{H}\right), 3.23-3.28(\mathrm{~m}, 1 \mathrm{H}), 3.32(\mathrm{~d}, J=13.6 \mathrm{~Hz}, 1 \mathrm{H})$, $3.47(\mathrm{~d}, J=13.6 \mathrm{~Hz}, 1 \mathrm{H}), 6.65(\mathrm{~d}, J=7.2 \mathrm{~Hz}, 1 \mathrm{H}), 6.86-6.92(\mathrm{~m}, 2 \mathrm{H}), 7.05-7.07(\mathrm{~m}, 1 \mathrm{H}), 7.18-7.25(\mathrm{~m}$, 2H), 7.38-7.40 (m, 1H), 7.49-7.52 (m, 1H); ${ }^{13} \mathrm{C}-\mathrm{NMR}\left(\mathrm{CDCl}_{3}\right)$ 8: 15.4, 42.2, 61.2, 82.7, 110.5, 122.6, 125.6, 126.5, 126.7, 126.9, 128.6, 129.7, 132.4, 132.9, 134.7, 140.9, 179.4; HRMS (ESI-TOF) $m / z$ : Calcd. for $\mathrm{C}_{17} \mathrm{H}_{16} \mathrm{BrNNaO}_{2}[\mathrm{M}+\mathrm{Na}]^{+}$: 368.0262; Found: 368.0262 .<smiles>CCOC1(Cc2ccccc2Cl)C(=O)Nc2ccccc21</smiles>

3-(2-Chlorobenzyl)-3-ethoxyindolin-2-one (3fb). Light orange solid, m.p. 207.1-209.2 ${ }^{\circ} \mathrm{C}$; yield 90\%; ${ }^{1} \mathrm{H}-\mathrm{NMR}\left(\right.$ DMSO- $\left.d_{6}\right) \delta: 1.01-1.05(\mathrm{~m}, 3 \mathrm{H}), 2.91-2.95(\mathrm{~m}, 1 \mathrm{H}), 3.05-3.08(\mathrm{~m}, 1 \mathrm{H}), 3.14(\mathrm{~d}, J=12.8 \mathrm{~Hz}, 1 \mathrm{H})$, $3.32(\mathrm{~d}, J=12.8 \mathrm{~Hz}, 1 \mathrm{H}), 6.72(\mathrm{~d}, J=7.8 \mathrm{~Hz}, 1 \mathrm{H}), 6.80-6.87(\mathrm{~m}, 2 \mathrm{H}), 7.14-7.28(\mathrm{~m}, 5 \mathrm{H}), 10.5(\mathrm{br} \mathrm{s}, 1 \mathrm{H})$; ${ }^{13}$ C-NMR (DMSO- $d_{6}$ ) $\delta: 15.3,40.1,60.0,82.0,109.8,121.5,124.8,126.5,128.6,129.0,129.7,132.2,132.5$, 142.0, 176.7; HRMS (ESI-TOF) $m / z$ : Calcd. for $\mathrm{C}_{17} \mathrm{H}_{16} \mathrm{ClNNaO}_{2}[\mathrm{M}+\mathrm{Na}]^{+}: 324.0767$; Found: 324.0769. 


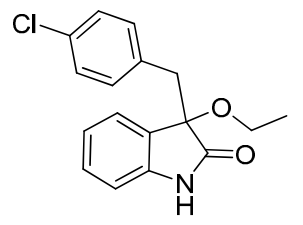

3-(4-Chlorobenzyl)-3-ethoxyindolin-2-one (3gb). Light orange solid, m.p. $154.1-156.2{ }^{\circ} \mathrm{C}$; yield 91\%; ${ }^{1} \mathrm{H}-\mathrm{NMR}\left(\mathrm{CDCl}_{3}\right) \delta: 1.14-1.18(\mathrm{~m}, 3 \mathrm{H}), 3.06(\mathrm{~d}, J=12.8 \mathrm{~Hz}, 1 \mathrm{H}), 3.11-3.15(\mathrm{~m}, 1 \mathrm{H}), 3.21-3.25(\mathrm{~m}, 1 \mathrm{H})$, $3.28(\mathrm{~d}, J=12.8 \mathrm{~Hz}, 1 \mathrm{H}), 6.81(\mathrm{~d}, J=8.0 \mathrm{~Hz}, 1 \mathrm{H}), 6.88(\mathrm{~d}, J=8.4 \mathrm{~Hz}, 2 \mathrm{H}), 7.02-7.05(\mathrm{~m}, 4 \mathrm{H}), 7.22-7.27$ $(\mathrm{m}, 1 \mathrm{H}), 9.04(\mathrm{br} \mathrm{s}, 1 \mathrm{H}) ;{ }^{13} \mathrm{C}-\mathrm{NMR}\left(\mathrm{CDCl}_{3}\right) \delta: 15.3,43.1,61.3,83.3,110.4,122.7,125.1,126.9,127.7,129.8$, 131.9, 132.5, 132.7, 140.9, 178.7; HRMS (ESI-TOF) $m / z$ : Calcd. for $\mathrm{C}_{17} \mathrm{H}_{16} \mathrm{ClNNaO}_{2}[\mathrm{M}+\mathrm{Na}]^{+}:$324.0767; Found: 324.0766 .

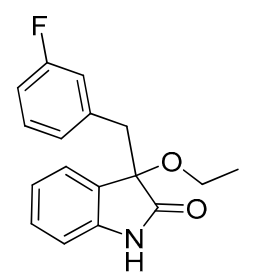

3-Ethoxy-3-(3-fluorobenzyl)indolin-2-one (3hb). Light orange solid, m.p. $118.3-119.8^{\circ} \mathrm{C}$; yield 89\%; ${ }^{1} \mathrm{H}-\mathrm{NMR}\left(\mathrm{CDCl}_{3}, 500 \mathrm{MHz}\right) \delta: 1.16-1.19(\mathrm{~m}, 3 \mathrm{H}), 3.08(\mathrm{~d}, J=13.0 \mathrm{~Hz}, 1 \mathrm{H}), 3.12-3.15(\mathrm{~m}, 1 \mathrm{H}), 3.24-3.27$ $(\mathrm{m}, 1 \mathrm{H}), 3.31(\mathrm{~d}, J=13.0 \mathrm{~Hz}, 1 \mathrm{H}), 6.69-6.75(\mathrm{~m}, 2 \mathrm{H}), 6.80-6.83(\mathrm{~m}, 2 \mathrm{H}), 7.00-7.05(\mathrm{~m}, 3 \mathrm{H}), 7.22-7.26(\mathrm{~m}$, 1H), 9.10 (br s, $1 \mathrm{H}) ;{ }^{13} \mathrm{C}-\mathrm{NMR}\left(\mathrm{CDCl}_{3}, 125 \mathrm{MHz}\right) \delta: 15.3,43.4,61.2,83.2,110.4,110.5,113.6,113.7,117.4$ $\left(\mathrm{d}, J_{C F}=21.3 \mathrm{~Hz}\right), 122.7,125.1,126.3,126.4,126.8,128.9\left(\mathrm{~d}, J_{C F}=8.8 \mathrm{~Hz}\right), 129.8,136.6\left(\mathrm{~d}, J_{C F}=7.5 \mathrm{~Hz}\right)$, $140.9,162.1\left(\mathrm{~d}, J_{C F}=243.8 \mathrm{~Hz}\right), 178.7$; HRMS (ESI-TOF) $m / z$ : Calcd. for $\mathrm{C}_{17} \mathrm{H}_{16} \mathrm{FNNaO}_{2}[\mathrm{M}+\mathrm{Na}]^{+}$: 308.1063; Found: 308.1067.

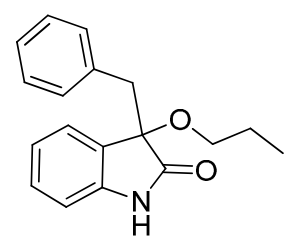

3-Benzyl-3-propoxyindolin-2-one (3ac). Light orange solid, m.p. $146.1-148.3{ }^{\circ} \mathrm{C}$; yield $86 \% ;{ }^{1} \mathrm{H}-\mathrm{NMR}$ $\left(\mathrm{CDCl}_{3}\right)$ ): 0.84-0.88 (m, 3H), 1.54-1.60 (m, 2H), 2.95-3.01 (m, 1H), $3.08(\mathrm{~d}, J=12.8 \mathrm{~Hz}, 1 \mathrm{H}), 3.15-3.20(\mathrm{~m}$, $1 \mathrm{H}), 3.33(\mathrm{~d}, J=12.8 \mathrm{~Hz}, 1 \mathrm{H}), 6.78(\mathrm{~d}, J=7.6 \mathrm{~Hz}, 1 \mathrm{H}), 6.96-7.13(\mathrm{~m}, 7 \mathrm{H}), 7.19-7.26(\mathrm{~m}, 1 \mathrm{H}), 8.90(\mathrm{br} \mathrm{s}, 1 \mathrm{H})$; ${ }^{13} \mathrm{C}-\mathrm{NMR}\left(\mathrm{CDCl}_{3}\right)$ 8: 10.5, 23.1, 43.8, 67.2, 83.4, 110.2, 122.5, 125.3, 126.7, 127.2, 127.6, 129.6, 130.7, 134.1, 140.9, 178.9; HRMS (ESI-TOF) $m / z$ : Calcd. for $\mathrm{C}_{18} \mathrm{H}_{19} \mathrm{NNaO}_{2}[\mathrm{M}+\mathrm{Na}]^{+}$: 304.1313; Found: 304.1315 .<smiles>CCCOC1(Cc2ccc(OC)cc2)C(=O)Nc2ccccc21</smiles>

3-(4-Methoxybenzyl)-3-propoxyindolin-2-one (3bc). Light orange solid, m.p. $145.5-146.9{ }^{\circ} \mathrm{C}$; yield 82\%; ${ }^{1} \mathrm{H}-\mathrm{NMR}\left(\mathrm{CDCl}_{3}\right)$ 8: 0.81-0.85 (m, 3H), 1.52-1.56 (m, 2H), 2.94-3.02 (m, 2H), 3.12-3.15 (m, 1H), $3.25(\mathrm{~d}$, $J=12.8 \mathrm{~Hz}, 1 \mathrm{H}), 3.66(\mathrm{~s}, 3 \mathrm{H}), 6.57-6.60(\mathrm{~m}, 2 \mathrm{H}), 6.78(\mathrm{~d}, J=7.6 \mathrm{~Hz}, 1 \mathrm{H}), 6.84-6.87(\mathrm{~m}, 2 \mathrm{H}), 6.97-7.00$ $(\mathrm{m}, 2 \mathrm{H}), 7.19-7.25(\mathrm{~m}, 1 \mathrm{H}), 8.99(\mathrm{br} \mathrm{s}, 1 \mathrm{H}) ;{ }^{13} \mathrm{C}-\mathrm{NMR}\left(\mathrm{CDCl}_{3}\right)$ 8: 10.7, 23.2, 43.0, 55.1, 67.3, 83.6, 110.4, 113.1, 122.6, 125.4, 126.2, 127.5, 129.7, 131.7, 141.2, 158.4, 179.3; HRMS (ESI-TOF) $m / z$ : Calcd. for $\mathrm{C}_{19} \mathrm{H}_{21} \mathrm{NNaO}_{3}[\mathrm{M}+\mathrm{Na}]^{+}$: 334.1419; Found: 334.1415. 


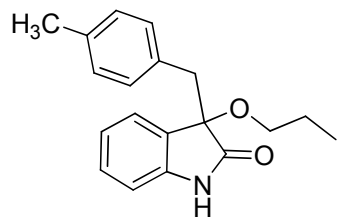

3-(4-Methylbenzyl)-3-propoxyindolin-2-one (3cc). Light orange solid, m.p. $75.1-76.8{ }^{\circ} \mathrm{C}$; yield 83\%; ${ }^{1} \mathrm{H}-\mathrm{NMR}\left(\mathrm{CDCl}_{3}\right) \delta: 0.76-0.80(\mathrm{~m}, 3 \mathrm{H}), 1.46-1.53(\mathrm{~m}, 2 \mathrm{H}), 2.15(\mathrm{~s}, 3 \mathrm{H}), 2.89-2.93(\mathrm{~m}, 1 \mathrm{H}), 2.98(\mathrm{~d}$, $J=12.8 \mathrm{~Hz}, 1 \mathrm{H}), 3.08-3.11(\mathrm{~m}, 1 \mathrm{H}), 3.21(\mathrm{~d}, J=12.8 \mathrm{~Hz}, 1 \mathrm{H}), 6.68(\mathrm{~d}, J=7.6 \mathrm{~Hz}, 1 \mathrm{H}), 6.76-6.82(\mathrm{~m}, 4 \mathrm{H})$, 6.92-6.97 (m, 2H), 7.12-7.19 (m, 1H), 8.41 (br s, 1H); ${ }^{13} \mathrm{C}-\mathrm{NMR}\left(\mathrm{CDCl}_{3}\right)$ 8: 10.5, 21.0, 23.1, 43.4, 67.2, 83.3, 110.1, 122.4, 125.3, 127.4, 128.3, 129.5, 130.5, 130.9, 136.1, 140.9, 178.7; HRMS (ESI-TOF) $m / z$ : Calcd. for $\mathrm{C}_{19} \mathrm{H}_{21} \mathrm{NNaO}_{2}[\mathrm{M}+\mathrm{Na}]^{+}$: 318.1470; Found: 318.1472 .<smiles>CCOC(=O)C1(Cc2ccc(Br)cc2)NC(=O)c2ccccc21</smiles>

3-(4-Bromobenzyl)-3-propoxyindolin-2-one (3dc). Light orange solid, m.p. $143.3-144.8^{\circ} \mathrm{C}$; yield 82\%;

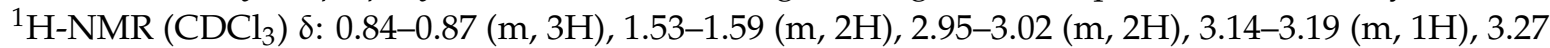
$(\mathrm{d}, J=12.8 \mathrm{~Hz}, 1 \mathrm{H}), 6.81-6.86(\mathrm{~m}, 3 \mathrm{H}), 6.94-6.96(\mathrm{~m}, 1 \mathrm{H}), 7.01-7.04(\mathrm{~m}, 1 \mathrm{H}), 7.20-7.24(\mathrm{~m}, 3 \mathrm{H}), 8.88$ (br s, $1 \mathrm{H}) ;{ }^{13} \mathrm{C}-\mathrm{NMR}\left(\mathrm{CDCl}_{3}\right)$ 8: 10.5, 23.0, 43.1, 67.2, 83.0, 110.4, 120.9, 122.6, 125.2, 126.9, 129.8, 130.7, 132.4, 133.2, 140.8, 178.7; HRMS (ESI-TOF) $m / z$ : Calcd. for $\mathrm{C}_{18} \mathrm{H}_{18} \mathrm{BrNNaO}_{2}[\mathrm{M}+\mathrm{Na}]^{+}$: 382.0419; Found: 382.0417.

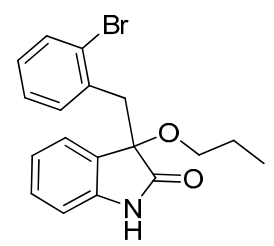

3-(2-Bromobenzyl)-3-propoxyindolin-2-one (3ec). Light orange solid, m.p. $153.1-154.9^{\circ} \mathrm{C}$; yield 81\%; ${ }^{1} \mathrm{H}-\mathrm{NMR}\left(\mathrm{CDCl}_{3}\right) \delta: 0.85-0.88(\mathrm{~m}, 3 \mathrm{H}), 1.54-1.62(\mathrm{~m}, 2 \mathrm{H}), 2.95(\mathrm{~d}, J=6.0 \mathrm{~Hz}, 1 \mathrm{H}), 3.21(\mathrm{~d}, J=6.4 \mathrm{~Hz}$, $1 \mathrm{H}), 3.32(\mathrm{~d}, J=11.2 \mathrm{~Hz}, 1 \mathrm{H}), 3.48(\mathrm{~d}, J=11.2 \mathrm{~Hz}, 1 \mathrm{H}), 6.59(\mathrm{~d}, J=6.0 \mathrm{~Hz}, 1 \mathrm{H}), 6.89-6.92(\mathrm{~m}, 2 \mathrm{H})$, 7.07-7.09 (m, 1H), 7.21-7.26 (m, 2H), $7.41(\mathrm{~d}, J=6.4 \mathrm{~Hz}, 1 \mathrm{H}), 7.56(\mathrm{~d}, J=6.0 \mathrm{~Hz}, 1 \mathrm{H}), 9.22(\mathrm{br} \mathrm{s}, 1 \mathrm{H})$; ${ }^{13} \mathrm{C}-\mathrm{NMR}\left(\mathrm{CDCl}_{3}\right)$ 8: 10.6, 23.1, 42.1, 67.0, 82.3, 110.3, 122.5, 125.5, 126.5, 126.6, 126.7, 128.4, 129.6, 132.3, 133.0, 134.7, 140.7, 179.2; HRMS (ESI-TOF) $m / z$ : Calcd. for $\mathrm{C}_{18} \mathrm{H}_{18} \mathrm{BrNNaO}_{2}[\mathrm{M}+\mathrm{Na}]^{+}$: 382.0419; Found: 382.0421 .

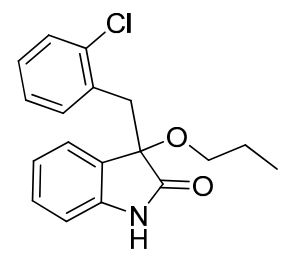

3-(2-Chlorobenzyl)-3-propoxyindolin-2-one (3fc). Light orange solid, m.p. $160.3-161.9^{\circ} \mathrm{C}$; yield 80\%; ${ }^{1} \mathrm{H}-\mathrm{NMR}\left(\mathrm{CDCl}_{3}\right)$ 8: 0.85-0.88 (m, 3H), 1.56-1.62 (m, 2H), 2.94-2.98 (m, 1H), 3.18-3.22 (m, 1H), 3.33 $(\mathrm{d}, J=13.6 \mathrm{~Hz}, 1 \mathrm{H}), 3.46(\mathrm{~d}, J=13.6 \mathrm{~Hz}, 1 \mathrm{H}), 6.69(\mathrm{~d}, J=7.2 \mathrm{~Hz}, 1 \mathrm{H}), 6.86-6.94(\mathrm{~m}, 2 \mathrm{H}), 7.13-7.26$ $(\mathrm{m}, 4 \mathrm{H}), 7.51(\mathrm{~d}, J=4.0 \mathrm{~Hz}, 1 \mathrm{H}), 9.15(\mathrm{br} \mathrm{s}, 1 \mathrm{H}) ;{ }^{13} \mathrm{C}-\mathrm{NMR}\left(\mathrm{CDCl}_{3}\right) \delta: 10.6,23.1,39.6,67.1,82.4,110.2$, $122.5,125.4,126.0,126.7,128.2,129.0,129.6,132.8,135.3,140.8,179.2$; HRMS (ESI-TOF) $m / z$ : Calcd. for $\mathrm{C}_{18} \mathrm{H}_{18} \mathrm{ClNNaO}_{2}[\mathrm{M}+\mathrm{Na}]^{+}$: 338.0924; Found: 338.0924. 


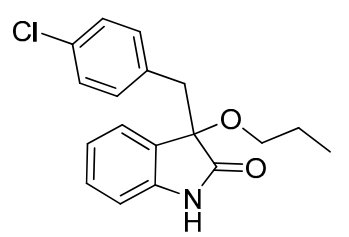

3-(4-Chlorobenzyl)-3-propoxyindolin-2-one (3gc). Light orange solid, m.p. 158.1-161.2 ${ }^{\circ} \mathrm{C}$; yield 84\%; ${ }^{1} \mathrm{H}-\mathrm{NMR}\left(\mathrm{CDCl}_{3}\right)$ 8: 0.84-0.87 (m, 3H), 1.53-1.60 (m, 2H), 2.96-3.00 (m, $\left.1 \mathrm{H}\right), 3.03(\mathrm{~d}, J=12.8 \mathrm{~Hz}, 1 \mathrm{H})$, $3.14-3.18(\mathrm{~m}, 1 \mathrm{H}), 3.29(\mathrm{~d}, J=12.8 \mathrm{~Hz}, 1 \mathrm{H}), 6.81(\mathrm{~d}, J=7.6 \mathrm{~Hz}, 1 \mathrm{H}), 6.89-6.97(\mathrm{~m}, 3 \mathrm{H}), 7.01-7.07(\mathrm{~m}$, $3 \mathrm{H}), 7.22-7.26(\mathrm{~m}, 1 \mathrm{H}), 8.90$ (br s, $1 \mathrm{H}) ;{ }^{13} \mathrm{C}-\mathrm{NMR}\left(\mathrm{CDCl}_{3}\right)$ 8: 10.5, 23.0, 43.1, 67.3, 83.0, 110.3, 122.6, $125.2,126.9,127.7,129.8,132.0,132.7,140.8,178.7$; HRMS (ESI-TOF) $m / z$ : Calcd. for $\mathrm{C}_{18} \mathrm{H}_{18} \mathrm{ClNNaO}_{2}$ $[\mathrm{M}+\mathrm{Na}]^{+}:$338.0924; Found: 338.0927.<smiles>CC(C)OC1(Cc2ccc(Br)cc2)C(=O)Nc2ccccc21</smiles>

3-(4-Bromobenzyl)-3-isopropoxyindolin-2-one (3dd). Light orange solid, m.p. 148.1-150.1 ${ }^{\circ} \mathrm{C}$; yield 77\%; ${ }^{1} \mathrm{H}-\mathrm{NMR}\left(\mathrm{CDCl}_{3}, 500 \mathrm{MHz}\right) \delta: 1.01(\mathrm{~d}, J=6.5 \mathrm{~Hz}, 3 \mathrm{H}), 1.10(\mathrm{~d}, J=6.0 \mathrm{~Hz}, 3 \mathrm{H}), 2.97(\mathrm{~d}, J=13.0 \mathrm{~Hz}$, $1 \mathrm{H}), 3.23(\mathrm{~d}, J=13.0 \mathrm{~Hz}, 1 \mathrm{H}), 6.78-6.83(\mathrm{~m}, 3 \mathrm{H}), 6.98-7.02(\mathrm{~m}, 2 \mathrm{H}), 7.19-7.27(\mathrm{~m}, 3 \mathrm{H}), 8.57(\mathrm{br} \mathrm{s}, 1 \mathrm{H})$; ${ }^{13} \mathrm{C}-\mathrm{NMR}\left(\mathrm{CDCl}_{3}, 125 \mathrm{MHz}\right)$ 8: 23.1, 24.1, 43.8, 69.5, 82.7, 110.3, 120.9, 122.4, 125.6, 127.3, 129.8, 130.6, 132.4, 133.2, 140.6, 179.3; HRMS (ESI-TOF) $m / z$ : Calcd. for $\mathrm{C}_{18} \mathrm{H}_{18} \mathrm{BrNNaO}_{2}[\mathrm{M}+\mathrm{Na}]^{+}: 382.0419$; Found: 382.0419 .<smiles>CC(C)OC1(Cc2ccccc2Br)C(=O)Nc2ccccc21</smiles>

3-(2-Bromobenzyl)-3-isopropoxyindolin-2-one (3ed). Light orange solid, m.p. 182.0-183.3 ${ }^{\circ} \mathrm{C}$; yield 74\%; ${ }^{1} \mathrm{H}-\mathrm{NMR}\left(\mathrm{CDCl}_{3}\right) \delta: 0.99(\mathrm{~d}, J=6.0 \mathrm{~Hz}, 3 \mathrm{H}), 1.10(\mathrm{~d}, J=6.0 \mathrm{~Hz}, 3 \mathrm{H}), 3.24(\mathrm{~d}, J=14.0 \mathrm{~Hz}, 1 \mathrm{H}), 3.40-3.44$ $(\mathrm{m}, 2 \mathrm{H}), 6.53(\mathrm{~d}, J=7.2 \mathrm{~Hz}, 1 \mathrm{H}), 6.84-6.89(\mathrm{~m}, 2 \mathrm{H}), 7.03-7.08(\mathrm{~m}, 1 \mathrm{H}), 7.18-7.25(\mathrm{~m}, 2 \mathrm{H}), 7.37-7.41(\mathrm{~m}$, 1H), 7.55-7.57 (m, 1H), 9.16 (br s, 1H); ${ }^{13} \mathrm{C}-\mathrm{NMR}\left(\mathrm{CDCl}_{3}\right)$ 8: 23.2, 24.0, 42.7, 69.3, 82.1, 110.5, 122.4, $126.1,126.7,126.8,126.9,128.5,129.7,132.3,133.1,134.9,140.6,180.4$; HRMS (ESI-TOF) $m / z$ : Calcd. for $\mathrm{C}_{18} \mathrm{H}_{18} \mathrm{BrNNaO}_{2}[\mathrm{M}+\mathrm{Na}]^{+}$: 382.0419; Found: 382.0422 .<smiles>CC(C)OC1(Cc2ccccc2Cl)C(=O)Nc2ccccc21</smiles>

3-(2-Chlorobenzyl)-3-isopropoxyindolin-2-one (3fd). Light orange solid, m.p. 197.2-198.7 ${ }^{\circ} \mathrm{C}$; yield 70\%; ${ }^{1} \mathrm{H}-\mathrm{NMR}\left(\mathrm{CDCl}_{3}\right) \delta: 1.01(\mathrm{~d}, J=6.0 \mathrm{~Hz}, 3 \mathrm{H}), 1.12(\mathrm{~d}, J=6.0 \mathrm{~Hz}, 3 \mathrm{H}), 3.27(\mathrm{~d}, J=13.6 \mathrm{~Hz}, 1 \mathrm{H}), 3.40-3.45$ $(\mathrm{m}, 2 \mathrm{H}), 6.66(\mathrm{~d}, J=7.2 \mathrm{~Hz}, 1 \mathrm{H}), 6.87-6.92(\mathrm{~m}, 2 \mathrm{H}), 7.12-7.26(\mathrm{~m}, 4 \mathrm{H}), 7.49-7.52(\mathrm{~m}, 1 \mathrm{H}), 9.16(\mathrm{br} \mathrm{s}$, $1 \mathrm{H}) ;{ }^{13} \mathrm{C}-\mathrm{NMR}\left(\mathrm{CDCl}_{3}\right)$ 8: 23.1, 24.0, 40.1, 69.2, 82.1, 110.3, 122.2, 125.9, 126.0, 127.0, 128.1, 128.9, 129.6, 132.9, 135.4, 140.5, 180.1; HRMS (ESI-TOF) $m / z$ : Calcd. for $\mathrm{C}_{18} \mathrm{H}_{18} \mathrm{ClNNaO}_{2}[\mathrm{M}+\mathrm{Na}]^{+}$: 338.0924; Found: 338.0925 . 


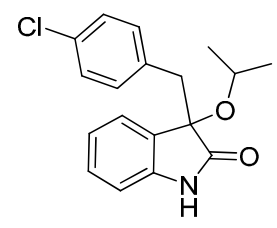

3-(4-Chlorobenzyl)-3-isopropoxyindolin-2-one (3gd). Light orange solid, m.p. 198.2-201.3 ${ }^{\circ} \mathrm{C}$; yield 72\%; ${ }^{1} \mathrm{H}-\mathrm{NMR}\left(\mathrm{CDCl}_{3}\right) \delta: 1.01(\mathrm{~d}, J=6.4 \mathrm{~Hz}, 3 \mathrm{H}), 1.08(\mathrm{~d}, J=6.0 \mathrm{~Hz}, 3 \mathrm{H}), 2.98(\mathrm{~d}, J=12.8 \mathrm{~Hz}, 1 \mathrm{H}), 3.25$ $(\mathrm{d}, J=12.8 \mathrm{~Hz}, 1 \mathrm{H}), 3.38-3.45(\mathrm{~m}, 1 \mathrm{H}), 6.81(\mathrm{~d}, J=7.6 \mathrm{~Hz}, 1 \mathrm{H}), 6.86-6.89(\mathrm{~m}, 2 \mathrm{H}), 6.97-7.04(\mathrm{~m}, 4 \mathrm{H})$ 7.22-7.27 (m, 1H), 8.97 (br s, $1 \mathrm{H}),{ }^{13} \mathrm{C}-\mathrm{NMR}\left(\mathrm{CDCl}_{3}\right)$ 8: 23.1, 24.1, 43.7, 69.5, 82.8, 110.4, 122.4, 125.6, 127.3, 127.7, $129.8,132.0,132.6,132.7,140.6,179.6$; HRMS (ESI-TOF) $m / z$ : Calcd. for $\mathrm{C}_{18} \mathrm{H}_{18} \mathrm{ClNNaO}_{2}$ $[\mathrm{M}+\mathrm{Na}]^{+}:$338.0924; Found: 338.0926 .<smiles>CCCCOC1(Cc2ccccc2)C(=O)Nc2ccccc21</smiles>

3-Benzyl-3-butoxyindolin-2-one (3ae). Light orange solid, m.p. $125.5-126.7{ }^{\circ} \mathrm{C}$; yield $80 \%$; ${ }^{1} \mathrm{H}-\mathrm{NMR}$ $\left(\mathrm{CDCl}_{3}\right)$ 8: $0.82-0.85(\mathrm{~m}, 3 \mathrm{H}), 1.28-1.36(\mathrm{~m}, 2 \mathrm{H}), 1.49-1.54(\mathrm{~m}, 2 \mathrm{H}), 3.01-3.09(\mathrm{~m}, 2 \mathrm{H}), 3.16-3.20(\mathrm{~m}$, $1 \mathrm{H}), 3.32(\mathrm{~d}, J=12.8 \mathrm{~Hz}, 1 \mathrm{H}), 6.78-6.81(\mathrm{~m}, 1 \mathrm{H}), 6.95-7.11(\mathrm{~m}, 7 \mathrm{H}), 7.19-7.26(\mathrm{~m}, 1 \mathrm{H}), 9.01(\mathrm{br} \mathrm{s}, 1 \mathrm{H})$; ${ }^{13} \mathrm{C}-\mathrm{NMR}\left(\mathrm{CDCl}_{3}\right)$ ): 13.8, 19.1, 31.8, 43.8, 65.3, 83.4, 110.3, 122.4, 125.2, 126.7, 127.2, 127.5, 129.6, 130.6, 134.1, 141.0, 179.1; HRMS (ESI-TOF) $m / z$ : Calcd. for $\mathrm{C}_{19} \mathrm{H}_{21} \mathrm{NNaO}_{2}[\mathrm{M}+\mathrm{Na}]^{+}$: 318.1470; Found: 318.1473 .

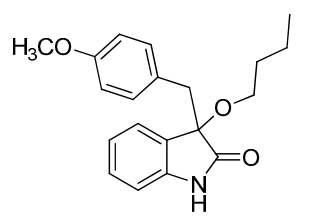

3-Butoxy-3-(4-methoxybenzyl)indolin-2-one (3be). Light orange solid, m.p. $139.5-141.7^{\circ} \mathrm{C}$; yield 83\%; ${ }^{1} \mathrm{H}-\mathrm{NMR}\left(\mathrm{CDCl}_{3}\right) \delta: 0.81-0.85(\mathrm{~m}, 3 \mathrm{H}), 1.28-1.34(\mathrm{~m}, 2 \mathrm{H}), 1.48-1.52(\mathrm{~m}, 2 \mathrm{H}), 6.59(\mathrm{~d}, J=8.4 \mathrm{~Hz}, 2 \mathrm{H}), 6.80$ $(\mathrm{d}, J=7.6 \mathrm{~Hz}, 1 \mathrm{H}), 6.87(\mathrm{~d}, J=8.8 \mathrm{~Hz}, 2 \mathrm{H}), 6.97-7.04(\mathrm{~m}, 2 \mathrm{H}), 7.20-7.24(\mathrm{~m}, 1 \mathrm{H}), 9.08(\mathrm{br} \mathrm{s}, 1 \mathrm{H}) ;{ }^{13} \mathrm{C}-\mathrm{NMR}$ $\left(\mathrm{CDCl}_{3}\right)$ 8: 13.8, 19.1, 31.8, 42.9, 54.9, 65.2, 83.5, 110.3, 112.9, 122.4, 125.2, 126.1, 127.3, 129.5, 131.5, 141.0, 158.2, 179.1; HRMS (ESI-TOF) $m / z$ : Calcd. for $\mathrm{C}_{20} \mathrm{H}_{23} \mathrm{NNaO}_{3}[\mathrm{M}+\mathrm{Na}]^{+}$: 348.1576; Found:348.1576.

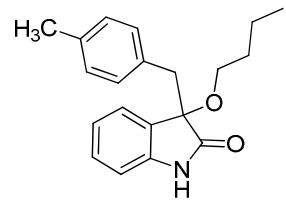

3-Butoxy-3-(4-methylbenzyl)indolin-2-one (3ce). Light orange solid, m.p. $105.1-107.7^{\circ} \mathrm{C}$; yield 85\%; ${ }^{1} \mathrm{H}-\mathrm{NMR}\left(\mathrm{CDCl}_{3}\right) \delta: 0.74-0.78(\mathrm{~m}, 3 \mathrm{H}), 1.22-1.25(\mathrm{~m}, 2 \mathrm{H}), 1.43-1.48(\mathrm{~m}, 2 \mathrm{H}), 2.13(\mathrm{~s}, 3 \mathrm{H}), 2.93-2.98(\mathrm{~m}$, $2 \mathrm{H}), 3.10-3.13(\mathrm{~m}, 1 \mathrm{H}), 3.20(\mathrm{~d}, J=13.2 \mathrm{~Hz}, 1 \mathrm{H}), 6.71(\mathrm{~d}, J=8.0 \mathrm{~Hz}, 1 \mathrm{H}), 6.75-6.81(\mathrm{~m}, 4 \mathrm{H}), 6.90-6.94$ $(\mathrm{m}, 2 \mathrm{H}), 7.12-7.18(\mathrm{~m}, 1 \mathrm{H}), 8.87(\mathrm{br} \mathrm{s}, 1 \mathrm{H}) ;{ }^{13} \mathrm{C}-\mathrm{NMR}\left(\mathrm{CDCl}_{3}\right)$ 8: 13.8, 19.1, 21.0, 31.9, 43.3, 65.2, 83.4, 110.2, 122.4, 125.2, 127.3, 128.2, 129.5, 130.5, 130.9, 136.1, 141.0, 179.0; HRMS (ESI-TOF) $m / z$ : Calcd. for $\mathrm{C}_{20} \mathrm{H}_{23} \mathrm{NNaO}_{2}[\mathrm{M}+\mathrm{Na}]^{+}:$332.1626; Found:332.1629. 


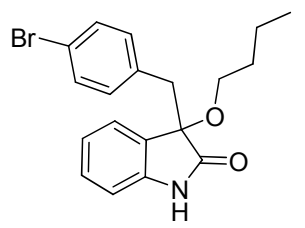

3-(4-Bromobenzyl)-3-butoxyindolin-2-one (3de). Light orange solid, m.p. $136.0-138.1{ }^{\circ} \mathrm{C}$; yield 84\%; ${ }^{1} \mathrm{H}-\mathrm{NMR}\left(\mathrm{CDCl}_{3}\right)$ 8: 0.82-0.85 (m, 3H), 1.30-1.35 (m, 2H), 1.48-1.54 (m, 2H), 2.99-3.03 (m, 2H), 3.17-3.19 $(\mathrm{m}, 1 \mathrm{H}), 3.27(\mathrm{~d}, J=13.0 \mathrm{~Hz}, 1 \mathrm{H}), 6.80-6.86(\mathrm{~m}, 3 \mathrm{H}), 6.94-6.96(\mathrm{~m}, 1 \mathrm{H}), 7.01-7.04(\mathrm{~m}, 1 \mathrm{H}), 7.20-7.27(\mathrm{~m}$, 3H), 8.93 (br s, $1 \mathrm{H}) ;{ }^{13} \mathrm{C}-\mathrm{NMR}\left(\mathrm{CDCl}_{3}\right) \delta: 13.8,19.1,31.8,43.1,65.3,83.0,110.4,120.9,122.6,125.2,126.9$, 129.8, 130.7, 132.4, 133.2, 140.8, 178.7; HRMS (ESI-TOF) $m / z$ : Calcd. for $\mathrm{C}_{19} \mathrm{H}_{20} \mathrm{BrNNaO}_{2}\left[\mathrm{M}+\mathrm{Na}^{+}\right.$: 396.0575; Found: 396.0578.

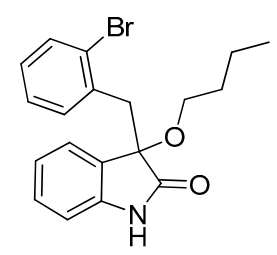

3-(2-Bromobenzyl)-3-butoxyindolin-2-one (3ee). Light orange solid, m.p. 98.9-99.3 ${ }^{\circ} \mathrm{C}$; yield 80\%; ${ }^{1} \mathrm{H}-\mathrm{NMR}$ $\left(\mathrm{CDCl}_{3}\right)$ 8: 0.82-0.85 (m, 3H), 1.25-1.36 (m, 2H), 1.45-1.51 (m, 2H), 3.00-3.01 (m, 1H), 3.22-3.24 (m, 1H), $3.32(\mathrm{~d}, J=11.2 \mathrm{~Hz}, 1 \mathrm{H}), 3.48(\mathrm{~d}, J=11.2 \mathrm{~Hz}, 1 \mathrm{H}), 6.59(\mathrm{~d}, J=6.0 \mathrm{~Hz}, 1 \mathrm{H}), 6.89-6.92(\mathrm{~m}, 2 \mathrm{H}), 7.07-7.08$ $(\mathrm{m}, 1 \mathrm{H}), 7.20-7.25(\mathrm{~m}, 2 \mathrm{H}), 7.40-7.42(\mathrm{~m}, 1 \mathrm{H}), 7.55(\mathrm{~d}, J=6.0 \mathrm{~Hz}, 1 \mathrm{H}), 9.55(\mathrm{br} \mathrm{s}, 1 \mathrm{H}) ;{ }^{13} \mathrm{C}-\mathrm{NMR}\left(\mathrm{CDCl}_{3}\right)$ $\delta: 13.8,19.2,31.8,42.1,65.1,82.4,110.4,122.4,125.4,126.5,126.6,126.7,128.4,129.5,132.3,132.9,134.7$, 140.8, 179.4; HRMS (ESI-TOF) $m / z$ : Calcd. for $\mathrm{C}_{19} \mathrm{H}_{20} \mathrm{BrNNaO}_{2}[\mathrm{M}+\mathrm{Na}]^{+}:$396.0575; Found: 396.0575 .<smiles>CCCCOC1(Cc2ccccc2Cl)C(=O)Nc2ccccc21</smiles>

3-Butoxy-3-(2-chlorobenzyl)indolin-2-one (3fe). Light orange solid, m.p. $142.1-144.0{ }^{\circ} \mathrm{C}$; yield $81 \%$; ${ }^{1} \mathrm{H}-\mathrm{NMR}\left(\mathrm{CDCl}_{3}\right) \delta: 0.82-0.86(\mathrm{~m}, 3 \mathrm{H}), 1.33-1.38(\mathrm{~m}, 2 \mathrm{H}), 1.49-1.57(\mathrm{~m}, 2 \mathrm{H}), 2.97-3.03(\mathrm{~m}, 1 \mathrm{H}), 3.20-3.25$ $(\mathrm{m}, 1 \mathrm{H}), 3.32(\mathrm{~d}, J=13.6 \mathrm{~Hz}, 1 \mathrm{H}), 3.46(\mathrm{~d}, J=13.6 \mathrm{~Hz}, 1 \mathrm{H}), 6.69(\mathrm{~d}, J=7.6 \mathrm{~Hz}, 1 \mathrm{H}), 6.87-6.94(\mathrm{~m}, 2 \mathrm{H})$, 7.13-7.26 (m, 4H), 7.47-7.50 (m, 1H), 9.29 (br s, $1 \mathrm{H}) ;{ }^{13} \mathrm{C}-\mathrm{NMR}\left(\mathrm{CDCl}_{3}\right)$ 8: 13.8, 19.2, 31.8, 39.6, 65.1, 82.5, $110.3,122.5,125.4,126.0,126.7,128.2,129.0,129.6,132.8,132.9,135.3,140.7,179.2$; HRMS (ESI-TOF) $m / z$ : Calcd. for $\mathrm{C}_{19} \mathrm{H}_{20} \mathrm{ClNNaO}_{2}[\mathrm{M}+\mathrm{Na}]^{+}$: 352.1080; Found:352.1082.<smiles>CCCCOC1(Cc2ccc(Cl)cc2)C(=O)Nc2ccccc21</smiles>

3-Butoxy-3-(4-chlorobenzyl)indolin-2-one (3ge). Light orange solid, m.p. $142.1-144.3{ }^{\circ} \mathrm{C}$; yield 83\%; ${ }^{1} \mathrm{H}-\mathrm{NMR}\left(\mathrm{CDCl}_{3}\right) \delta: 0.81-0.85(\mathrm{~m}, 3 \mathrm{H}), 1.30-1.34(\mathrm{~m}, 2 \mathrm{H}), 1.50-1.54(\mathrm{~m}, 2 \mathrm{H}), 3.00-3.05(\mathrm{~m}, 2 \mathrm{H}), 3.16-3.21$ (m, 1H), $3.28(\mathrm{~d}, J=12.8 \mathrm{~Hz}, 1 \mathrm{H}), 6.80-6.83(\mathrm{~m}, 1 \mathrm{H}), 6.89-6.96(\mathrm{~m}, 3 \mathrm{H}), 7.01-7.06(\mathrm{~m}, 3 \mathrm{H}), 7.21-7.27$ (m, 1H), 9.09 (br s, $1 \mathrm{H}) ;{ }^{13} \mathrm{C}-\mathrm{NMR}\left(\mathrm{CDCl}_{3}\right)$ 8: 13.7, 19.1, 31.8, 43.1, 65.3, 83.1, 110.4, 122.6, 125.1, 126.9, $127.7,129.7,132.0,132.7,140.9,178.8$; HRMS (ESI-TOF) $m / z$ : Calcd. for $\mathrm{C}_{19} \mathrm{H}_{20} \mathrm{ClNNaO}_{2}[\mathrm{M}+\mathrm{Na}]^{+}$: 352.1080; Found:352.1083. 


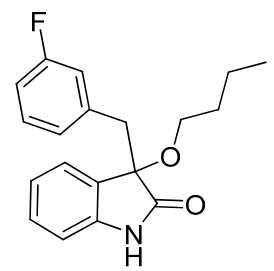

3-Butoxy-3-(3-fluorobenzyl)indolin-2-one (3he). Light orange solid, m.p. $129.0-131.1{ }^{\circ} \mathrm{C}$; yield $74 \%$; ${ }^{1} \mathrm{H}-\mathrm{NMR}\left(\mathrm{CDCl}_{3}, 500 \mathrm{MHz}\right) \delta: 0.82-0.86(\mathrm{~m}, 3 \mathrm{H}), 1.26-1.35(\mathrm{~m}, 2 \mathrm{H}), 1.45-1.57(\mathrm{~m}, 2 \mathrm{H}), 3.02-3.05(\mathrm{~m}$, $2 \mathrm{H}), 3.19-3.22(\mathrm{~m}, 1 \mathrm{H}), 3.32(\mathrm{~d}, J=13.5 \mathrm{~Hz}, 1 \mathrm{H}), 6.72-6.76(\mathrm{~m}, 2 \mathrm{H}), 6.80-6.84(\mathrm{~m}, 2 \mathrm{H}), 6.91-6.95(\mathrm{~m}$, 1H), 7.01-7.05 (m, 2H), 7.22-7.26 (m, 1H), 9.18 (br s, 1H); ${ }^{13} \mathrm{C}-\mathrm{NMR}\left(\mathrm{CDCl}_{3}, 125 \mathrm{MHz}\right) \delta: 13.7,19.1$, 31.8, 43.4, 65.3, 83.0, 110.4, $113.6\left(\mathrm{~d}, J_{C F}=21.1 \mathrm{~Hz}\right), 117.4\left(\mathrm{~d}, J_{C F}=21.2 \mathrm{~Hz}\right), 122.6,125.1,126.4,126.9$, $128.8,128.9,129.8,136.7,136.8,140.9,162.1\left(\mathrm{~d}, J_{C F}=243.8 \mathrm{~Hz}\right), 178.9$; HRMS (ESI-TOF) $m / z$ : Calcd. for $\mathrm{C}_{19} \mathrm{H}_{20} \mathrm{FNNaO}_{2}[\mathrm{M}+\mathrm{Na}]^{+}$: 336.1376; Found:336.1377.<smiles>O=C1Nc2ccccc2C1(O)Cc1ccccc1</smiles>

3-Benzyl-3-(benzyloxy)indolin-2-one (3af). Light orange solid, m.p. $168.8-170.3{ }^{\circ} \mathrm{C}$; yield $84 \%$; ${ }^{1} \mathrm{H}-\mathrm{NMR}$ $\left(\mathrm{CDCl}_{3}\right) \delta: 3.18(\mathrm{~d}, J=12.8 \mathrm{~Hz}, 1 \mathrm{H}), 3.41(\mathrm{~d}, J=12.8 \mathrm{~Hz}, 1 \mathrm{H}), 4.12(\mathrm{~d}, J=8.2 \mathrm{~Hz}, 1 \mathrm{H}), 4.25(\mathrm{~d}, J=12.8 \mathrm{~Hz}$, $1 \mathrm{H}), 6.80(\mathrm{~d}, \mathrm{~J}=8.0 \mathrm{~Hz}, 1 \mathrm{H}), 6.97-7.10(\mathrm{~m}, 7 \mathrm{H}), 7.21-7.29(\mathrm{~m}, 6 \mathrm{H}), 8.97(\mathrm{br} \mathrm{s}, 1 \mathrm{H}) ;{ }^{13} \mathrm{C}-\mathrm{NMR}\left(\mathrm{CDCl}_{3}\right) \delta$ : 43.8, 67.8, 83.8, 110.4, 122.6, 125.4, 126.8, 127.6, 127.7, 127.8, 128.2, 129.9, 130.7, 133.9, 137.5, 141.1, 178.4; HRMS (ESI-TOF) $m / z$ : Calcd. for $\mathrm{C}_{22} \mathrm{H}_{19} \mathrm{NNaO}_{2}[\mathrm{M}+\mathrm{Na}]^{+}$: 352.1313; Found:352.1313.<smiles>COc1ccc(CC2(OCc3ccccc3)C(=O)Nc3ccccc32)cc1</smiles>

3-(Benzyloxy)-3-(4-methoxybenzyl)indolin-2-one (3bf). Light orange solid, m.p. 218.1-219.8 ${ }^{\circ} \mathrm{C}$; yield 85\%; ${ }^{1} \mathrm{H}-\mathrm{NMR}\left(\mathrm{CDCl}_{3}\right) \delta: 3.12(\mathrm{~d}, J=13.2 \mathrm{~Hz}, 1 \mathrm{H}), 3.34(\mathrm{~d}, J=13.2 \mathrm{~Hz}, 1 \mathrm{H}), 3.64(\mathrm{~s}, 3 \mathrm{H}), 4.11(\mathrm{~d}, J=10.8 \mathrm{~Hz}$, $1 \mathrm{H}), 4.23(\mathrm{~d}, J=10.4 \mathrm{~Hz}, 1 \mathrm{H}), 6.58-6.61(\mathrm{~m}, 2 \mathrm{H}), 6.79-6.81(\mathrm{~m}, 1 \mathrm{H}), 6.87-6.89(\mathrm{~m}, 2 \mathrm{H}), 7.05-7.10(\mathrm{~m}, 2 \mathrm{H})$, 7.22-7.29 (m, 6H), 8.88 (br s, $1 \mathrm{H}) ;{ }^{13} \mathrm{C}-\mathrm{NMR}\left(\mathrm{CDCl}_{3}\right)$ 8: 43.0, 55.1, 68.0, 84.0, 110.6, 113.2, 122.8, 125.5, 126.0, 127.1, 127.8, 128.0, 128.3, 130.0, 131.8, 137.7, 141.2, 158.5, 178.7; HRMS (ESI-TOF) $m / z$ : Calcd. for $\mathrm{C}_{23} \mathrm{H}_{21} \mathrm{NNaO}_{3}[\mathrm{M}+\mathrm{Na}]^{+}:$382.1419; Found:382.1421.<smiles>Cc1ccc(CC2(OCc3ccccc3)C(=O)Nc3ccccc32)cc1</smiles>

3-(Benzyloxy)-3-(4-methylbenzyl)indolin-2-one (3cf). Light orange solid, m.p. 132.2-134.3 ${ }^{\circ} \mathrm{C}$; yield 80\%; ${ }^{1} \mathrm{H}-\mathrm{NMR}\left(\mathrm{CDCl}_{3}\right) \delta: 2.10(\mathrm{~s}, 3 \mathrm{H}), 3.06(\mathrm{~d}, J=13.2 \mathrm{~Hz}, 1 \mathrm{H}), 3.28(\mathrm{~d}, J=12.8 \mathrm{~Hz}, 1 \mathrm{H}), 4.03(\mathrm{~d}, J=10.4 \mathrm{~Hz}$, $1 \mathrm{H}), 4.16(\mathrm{~d}, J=10.8 \mathrm{~Hz}, 1 \mathrm{H}), 6.72(\mathrm{~d}, J=8.0 \mathrm{~Hz}, 1 \mathrm{H}), 6.78(\mathrm{~s}, 4 \mathrm{H}), 6.96-7.02(\mathrm{~m}, 2 \mathrm{H}), 7.14-7.20(\mathrm{~m}, 6 \mathrm{H})$, 8.93 (br s, $1 \mathrm{H}) ;{ }^{13} \mathrm{C}-\mathrm{NMR}\left(\mathrm{CDCl}_{3}\right)$ 8: 21.0, 43.3, 67.8, 83.8, 110.4, 122.6, 125.3, 126.9, 127.6, 127.8, 128.2, 128.4, 129.8, 130.5, 130.7, 136.2, 137.6, 141.1, 178.5; HRMS (ESI-TOF) $m / z$ : Calcd. for $\mathrm{C}_{23} \mathrm{H}_{21} \mathrm{NNaO}_{2}$ $[\mathrm{M}+\mathrm{Na}]^{+}:$366.1470; Found:366.1474. 
<smiles>O=C1Nc2ccccc2C1(Cc1ccccc1)OCc1ccccc1</smiles>

3-(Benzyloxy)-3-(4-bromobenzyl)indolin-2-one (3df). Light orange solid, m.p. 201.2-203.2 ${ }^{\circ} \mathrm{C}$; yield 82\%; ${ }^{1} \mathrm{H}-\mathrm{NMR}\left(\mathrm{DMSO}-d_{6}\right) \delta: 3.08(\mathrm{~d}, J=10.0 \mathrm{~Hz}, 1 \mathrm{H}), 3.27(\mathrm{~d}, J=10.0 \mathrm{~Hz}, 1 \mathrm{H}), 3.99(\mathrm{~d}, J=8.4 \mathrm{~Hz}, 1 \mathrm{H}), 4.12$ $(\mathrm{d}, J=8.4 \mathrm{~Hz}, 1 \mathrm{H}), 6.71(\mathrm{~d}, J=6.0 \mathrm{~Hz}, 1 \mathrm{H}), 6.86(\mathrm{~d}, J=6.4 \mathrm{~Hz}, 1 \mathrm{H}), 7.19-7.22(\mathrm{~m}, 1 \mathrm{H}), 7.26-7.31(\mathrm{~m}$, 9H); ${ }^{13}$ C-NMR (DMSO- $d_{6}$ ) $\delta: ~ 41.4,66.1,82.3,109.5,119.5,121.4,124.5,125.6,127.0,127.1,127.7,129.5$, 130.0, 132.0, 133.2, 137.2, 141.8, 175.6; HRMS (ESI-TOF) $m / z$ : Calcd. for $\mathrm{C}_{22} \mathrm{H}_{18} \mathrm{BrNNaO}_{2}[\mathrm{M}+\mathrm{Na}]^{+}$: 430.0419; Found:430.0423.<smiles>O=C1Nc2ccccc2C1(Cc1ccccc1Br)OCc1ccccc1</smiles>

3-(Benzyloxy)-3-(2-bromobenzyl)indolin-2-one (3ef). Light orange solid, m.p. $158.8-160.1{ }^{\circ} \mathrm{C}$; yield 81\%; ${ }^{1} \mathrm{H}-\mathrm{NMR}\left(\mathrm{CDCl}_{3}\right) \delta: 3.42(\mathrm{~d}, J=14.0 \mathrm{~Hz}, 1 \mathrm{H}), 3.58(\mathrm{~d}, J=14.0 \mathrm{~Hz}, 1 \mathrm{H}), 4.12(\mathrm{~d}, J=10.8 \mathrm{~Hz}, 1 \mathrm{H}), 4.30(\mathrm{~d}$, $J=10.8 \mathrm{~Hz}, 1 \mathrm{H}), 6.70(\mathrm{~d}, J=7.6 \mathrm{~Hz}, 1 \mathrm{H}), 6.91-6.95(\mathrm{~m}, 2 \mathrm{H}), 7.07-7.09(\mathrm{~m}, 1 \mathrm{H}), 7.21-7.30(\mathrm{~m}, 7 \mathrm{H}), 7.42(\mathrm{~d}$, $J=8.1 \mathrm{~Hz}, 1 \mathrm{H}), 7.56(\mathrm{~d}, J=8.0 \mathrm{~Hz}, 1 \mathrm{H}), 9.35(\mathrm{br} \mathrm{s}, 1 \mathrm{H}) ;{ }^{13} \mathrm{C}-\mathrm{NMR}\left(\mathrm{CDCl}_{3}\right) \delta: 42.2,67.8,82.9,110.7,122.8$, 125.8, 126.3, 126.6, 127.0, 127.7, 127.8, 128.3, 128.7, 130.0, 132.5, 133.1, 134.6, 137.8, 141.0, 179.0; HRMS (ESI-TOF) $m / z$ : Calcd. for $\mathrm{C}_{22} \mathrm{H}_{18} \mathrm{BrNNaO}_{2}[\mathrm{M}+\mathrm{Na}]^{+}: 430.0419$; Found:430.0418.<smiles>O=C1Nc2ccccc2C1(Cc1ccccc1Cl)OCc1ccccc1</smiles>

3-(Benzyloxy)-3-(2-chlorobenzyl)indolin-2-one (3ff). Light orange solid, m.p. $145.3-147.2{ }^{\circ} \mathrm{C}$; yield 82\%; ${ }^{1} \mathrm{H}-\mathrm{NMR}\left(\mathrm{CDCl}_{3}\right) \delta: 3.42(\mathrm{~d}, J=14.0 \mathrm{~Hz}, 1 \mathrm{H}), 3.56(\mathrm{~d}, J=13.6 \mathrm{~Hz}, 1 \mathrm{H}), 4.12(\mathrm{~d}, J=10.8 \mathrm{~Hz}, 1 \mathrm{H}), 4.29$ $(\mathrm{d}, J=10.8 \mathrm{~Hz}, 1 \mathrm{H}), 6.80(\mathrm{~d}, J=7.2 \mathrm{~Hz}, 1 \mathrm{H}), 6.89-6.96(\mathrm{~m}, 2 \mathrm{H}), 7.12-7.15(\mathrm{~m}, 2 \mathrm{H}), 7.20-7.28(\mathrm{~m}, 7 \mathrm{H})$, 7.48-7.50 (m, 1H), 9.34 (br s, 1H); ${ }^{13} \mathrm{C}-\mathrm{NMR}\left(\mathrm{CDCl}_{3}\right)$ 8: 39.6, 67.7, 82.9, 110.5, 122.6, 125.5, 126.1, 126.3, 127.6, 128.2, 128.3, 129.1, 129.9, 132.6, 132.9, 135.3, 137.6, 140.8, 178.7; HRMS (ESI-TOF) $m / z$ : Calcd. for $\mathrm{C}_{22} \mathrm{H}_{18} \mathrm{ClNNaO}_{2}[\mathrm{M}+\mathrm{Na}]^{+}$: 386.0924; Found:386.0925.<smiles>O=C1Nc2ccccc2C1(Cc1ccccc1)OCc1ccccc1</smiles>

3-(Benzyloxy)-3-(4-chlorobenzyl)indolin-2-one (3gf). Light orange solid, m.p. 110.3-112.8 ${ }^{\circ} \mathrm{C}$; yield 83\%; ${ }^{1} \mathrm{H}-\mathrm{NMR}\left(\mathrm{DMSO}-d_{6}\right) \delta: 3.09(\mathrm{~d}, J=12.8 \mathrm{~Hz}, 1 \mathrm{H}), 3.29(\mathrm{~d}, J=12.8 \mathrm{~Hz}, 1 \mathrm{H}), 3.99(\mathrm{~d}, J=10.8 \mathrm{~Hz}, 1 \mathrm{H})$, $4.13(\mathrm{~d}, J=10.8 \mathrm{~Hz}, 1 \mathrm{H}), 6.71(\mathrm{~d}, J=8.0 \mathrm{~Hz}, 1 \mathrm{H}), 6.92(\mathrm{~d}, J=8.4 \mathrm{~Hz}, 2 \mathrm{H}), 7.00-7.03(\mathrm{~m}, 1 \mathrm{H}), 7.15-7.30$ (m, 9H), 10.5 (br s, $1 \mathrm{H}) ;{ }^{13} \mathrm{C}-\mathrm{NMR}$ (DMSO- $d_{6}$ ) $\delta: 41.4,66.1,82.4,109.5,121.4,124.5,125.7,127.0,127.1$, 127.7, 129.5, 131.0, 131.6, 132.9, 137.2, 141.8, 175.6; HRMS (ESI-TOF) $m / z$ : Calcd. for $\mathrm{C}_{22} \mathrm{H}_{18} \mathrm{ClNNaO}_{2}$ $[\mathrm{M}+\mathrm{Na}]^{+}:$386.0924; Found:386.0924. 
<smiles>O=C1Nc2ccccc2C1(Cc1ccccc1)OCc1cccc(F)c1</smiles>

3-(Benzyloxy)-3-(3-fluorobenzyl)indolin-2-one (3hf). Light orange solid, m.p. $153.2-155.1{ }^{\circ} \mathrm{C}$; yield 75\%; ${ }^{1} \mathrm{H}-\mathrm{NMR}\left(\mathrm{CDCl}_{3}, 500 \mathrm{MHz}\right) \delta: 3.16(\mathrm{~d}, J=10.4 \mathrm{~Hz}, 1 \mathrm{H}), 3.39(\mathrm{~d}, J=13.4 \mathrm{~Hz}, 1 \mathrm{H}), 4.13(\mathrm{~d}, J=11.0 \mathrm{~Hz}$, $1 \mathrm{H}), 4.27(\mathrm{~d}, J=10.5 \mathrm{~Hz}, 1 \mathrm{H}), 6.72-6.74(\mathrm{~m}, 1 \mathrm{H}), 6.76-6.78(\mathrm{~m}, 1 \mathrm{H}), 6.81-6.84(\mathrm{~m}, 2 \mathrm{H}), 7.04-7.06(\mathrm{~m}$, $3 \mathrm{H}), 7.23-7.29(\mathrm{~m}, 6 \mathrm{H}) ;{ }^{13} \mathrm{C}-\mathrm{NMR}\left(\mathrm{CDCl}_{3}, 125 \mathrm{MHz}\right) \delta: 43.4,67.9,83.4,110.5,113.7\left(\mathrm{~d}, J_{\mathrm{CF}}=20.1 \mathrm{~Hz}\right)$, $117.4\left(\mathrm{~d}, J_{C F}=21.3 \mathrm{~Hz}\right), 122.8,125.3,126.5,127.7,127.8,128.3,129.0,129.1,130.1,136.5,137.4,141.0$, $162.1\left(\mathrm{~d}, J_{C F}=243.8 \mathrm{~Hz}\right), 178.2$; HRMS (ESI-TOF) $m / z$ : Calcd. for $\mathrm{C}_{22} \mathrm{H}_{18} \mathrm{FNNaO}_{2}[\mathrm{M}+\mathrm{Na}]^{+}: 370.1219$; Found: 370.1221 .<smiles>O=C1Nc2ccc(Cl)cc2C1(Cc1ccccc1)OCc1ccccc1</smiles>

3-Benzyl-3-(benzyloxy)-5-chloroindolin-2-one (3if). Light orange solid, m.p. $217.3-220.5^{\circ} \mathrm{C}$; yield 82\%; ${ }^{1} \mathrm{H}-\mathrm{NMR}\left(\mathrm{DMSO}-d_{6}\right) \delta: 3.09(\mathrm{~d}, J=12.4 \mathrm{H}, 1 \mathrm{H}), 3.33(\mathrm{~d}, J=12.8 \mathrm{~Hz}, 1 \mathrm{H}), 4.03(\mathrm{~d}, J=10.8 \mathrm{~Hz}, 1 \mathrm{H}), 4.16$ $(\mathrm{d}, J=10.8 \mathrm{~Hz}, 1 \mathrm{H}), 6.68(\mathrm{~d}, J=8.8 \mathrm{~Hz}, 1 \mathrm{H}), 6.92-6.95(\mathrm{~m}, 2 \mathrm{H}), 7.11-7.13(\mathrm{~m}, 3 \mathrm{H}), 7.22-7.32(\mathrm{~m}, 7 \mathrm{H})$, 10.6 (br s, $1 \mathrm{H}$ ) ${ }^{13} \mathrm{C}-\mathrm{NMR}$ (DMSO- $d_{6}$ ) $\delta: 42.4,66.8,83.2,111.3,125.2,125.9,126.8,127.5,127.6,127.7$, 128.2, 128.5, 129.7, 130.3, 133.9, 137.6, 141.2, 176.0; HRMS (ESI-TOF) $m / z$ : Calcd. for $\mathrm{C}_{22} \mathrm{H}_{18} \mathrm{ClNNaO}_{2}$ $[\mathrm{M}+\mathrm{Na}]^{+}:$386.0924; Found:386.0926.<smiles>O=C1Nc2cc(Cl)ccc2C1(Br)Cc1ccccc1</smiles>

3-Benzyl-3-(benzyloxy)-6-chloroindolin-2-one (3jf). Light orange solid, m.p. 165.1-168.5 ${ }^{\circ} \mathrm{C}$; yield 85\%; ${ }^{1} \mathrm{H}-\mathrm{NMR}\left(\mathrm{CDCl}_{3}\right) \delta: 3.04(\mathrm{~d}, J=13.2 \mathrm{~Hz}, 1 \mathrm{H}), 3.31(\mathrm{~d}, J=13.2 \mathrm{~Hz}, 1 \mathrm{H}), 4.01(\mathrm{~d}, J=10.8 \mathrm{~Hz}, 1 \mathrm{H}), 4.16$ $(\mathrm{d}, J=10.8 \mathrm{~Hz}, 1 \mathrm{H}), 6.75-6.76(\mathrm{~m}, 1 \mathrm{H}), 6.84-6.94(\mathrm{~m}, 4 \mathrm{H}), 7.00-7.06(\mathrm{~m}, 3 \mathrm{H}), 7.16-7.23(\mathrm{~m}, 5 \mathrm{H}), 9.04$ (br s, $1 \mathrm{H})$; ${ }^{13} \mathrm{C}-\mathrm{NMR}\left(\mathrm{CDCl}_{3}\right)$ $\delta: 43.6,67.9,83.4,111.2,122.7,125.1,126.4,127.0,127.8,128.3,130.7,133.6$, 135.5, 137.2, 142.1, 178.5; HRMS (ESI-TOF) $m / z$ : Calcd. for $\mathrm{C}_{22} \mathrm{H}_{18} \mathrm{ClNNaO}_{2}[\mathrm{M}+\mathrm{Na}]^{+}$: 386.0924; Found: 386.0925 .<smiles>Cc1ccc(CC2(OCCO)C(=O)Nc3ccccc32)cc1</smiles>

3-(2-Hydroxyethoxy)-3-(4-methylbenzyl)indolin-2-one (3cg). Light orange solid, m.p. 211.6-213.4 ${ }^{\circ} \mathrm{C}$; yield $83 \%{ }^{1}{ }^{1} \mathrm{H}-\mathrm{NMR}\left(\mathrm{DMSO}-d_{6}\right) \delta: 2.14(\mathrm{~s}, 3 \mathrm{H}), 2.95-3.03(\mathrm{~m}, 2 \mathrm{H}), 3.08-3.12(\mathrm{~m}, 1 \mathrm{H}), 3.17(\mathrm{~d}, J=10.0 \mathrm{~Hz}, 1 \mathrm{H})$, 3.38-3.42 (m, 2H), $4.57(\mathrm{br} \mathrm{s}, 1 \mathrm{H}), 6.61(\mathrm{~d}, J=6.4 \mathrm{~Hz}, 1 \mathrm{H}), 6.73(\mathrm{~d}, J=6.4 \mathrm{~Hz}, 2 \mathrm{H}), 6.86(\mathrm{~d}, J=6.0 \mathrm{~Hz}, 2 \mathrm{H})$, 6.96-6.99 (m, 1H), 7.14-7.17 (m, 1H), 7.21 (d, $J=5.6 \mathrm{~Hz}, 1 \mathrm{H}), 10.3(\mathrm{br} \mathrm{s}, 1 \mathrm{H}) ;{ }^{13} \mathrm{C}-\mathrm{NMR}\left(\mathrm{DMSO}-d_{6}\right) \delta$ : 20.1, 41.7, 59.6, 65.9, 82.5, 109.2, 121.2, 124.5, 126.3, 127.7, 129.2, 129.6, 130.6, 135.0, 141.8, 175.8; HRMS (ESI-TOF) $m / z$ : Calcd. for $\mathrm{C}_{18} \mathrm{H}_{19} \mathrm{NNaO}_{3}[\mathrm{M}+\mathrm{Na}]^{+}$: 320.1263; Found:320.1267. 


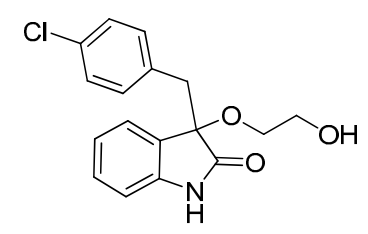

3-(4-Chlorobenzyl)-3-(2-hydroxyethoxy)indolin-2-one (3gg). Light orange solid, m.p. 205.3-207.3 ${ }^{\circ}$; yield 87\%; ${ }^{1} \mathrm{H}-\mathrm{NMR}\left(\mathrm{CDCl}_{3}\right) \delta: 2.93-2.97(\mathrm{~m}, 1 \mathrm{H}), 3.03(\mathrm{~d}, J=6.4 \mathrm{~Hz}, 1 \mathrm{H}), 3.07-3.11(\mathrm{~m}, 1 \mathrm{H}), 3.21$ $(\mathrm{d}, J=6.4 \mathrm{~Hz}, 1 \mathrm{H}), 3.39-3.43(\mathrm{~m}, 2 \mathrm{H}), 4.56-4.58(\mathrm{~m}, 1 \mathrm{H}), 6.64-6.65(\mathrm{~m}, 1 \mathrm{H}), 6.88(\mathrm{~d}, J=6.4 \mathrm{~Hz}, 2 \mathrm{H})$, 6.97-7.00 (m, 1H), 7.13-7.19 (m, 4H), 10.4 (br s, 1H); ${ }^{13} \mathrm{C}-\mathrm{NMR}\left(\mathrm{CDCl}_{3}\right)$ 8: 41.3, 59.5, 66.0, 82.2, 109.3, $121.3,124.5,125.9,127.1,129.4,130.9,131.5,132.9,141.7,175.7$; HRMS (ESI-TOF) $m / z$ : Calcd. for $\mathrm{C}_{17} \mathrm{H}_{16} \mathrm{ClNNaO}_{3}[\mathrm{M}+\mathrm{Na}]^{+}:$340.0716; Found:340.0716.<smiles>O=C1Nc2ccccc2C1(Cc1ccccc1)OCC(O)CO</smiles>

3-Benzyl-3-(2,3-dihydroxypropoxy)indolin-2-one (3ah). Light orange oil; yield 51\%, 1:1dr; ${ }^{1} \mathrm{H}-\mathrm{NMR}$ $\left(\mathrm{CDCl}_{3}\right)$ 8: 3.03-3.10 (m, 2H), 3.12-3.18 (m, 1H), 3.21-3.32 (m, 2.6 H), 3.45-3.49 (m, 1H), 3.59-3.84 $(\mathrm{m}, 2.6 \mathrm{H}), 6.74-6.77(\mathrm{~m}, 1 \mathrm{H}), 6.92-7.04(\mathrm{~m}, 4 \mathrm{H}), 7.07-7.15(\mathrm{~m}, 3 \mathrm{H}), 7.19-7.23(\mathrm{~m}, 1 \mathrm{H}), 8.65(\mathrm{br} \mathrm{s}, 1 \mathrm{H})$; ${ }^{13} \mathrm{C}-\mathrm{NMR}\left(\mathrm{CDCl}_{3}\right)$ 8: 43.6, 63.4, 63.5, 66.7, 67.7, 70.4, 70.7, 83.6, 83.7, 110.6, 110.7, 122.8, 126.9, 127.7, 130.0, 130.5, 130.6, 133.6, 140.8, 178.7, 178.8; HRMS (ESI-TOF) $m / z$ : Calcd. for $\mathrm{C}_{18} \mathrm{H}_{19} \mathrm{NNaO}_{4}[\mathrm{M}+\mathrm{Na}]^{+}$: 336.1212; Found:336.1215.

\section{Conclusions}

In conclusion, we have developed a highly efficient and eco-friendly method for the synthesis of multisubstituted 3-alkoxylated-2-oxindoles 3 via direct alkoxylation of 3-halooxindoles 1. A wide variety of multisubstituted 3-alkoxylated-2-oxindole scaffolds were obtained smoothly in good yields (up to $94 \%$ ) by simple heating in an oil bath at $30^{\circ} \mathrm{C}$ for $24 \mathrm{~h}$. A particularly valuable feature of this method was the development of environmentally-friendly chemistry using alcohols 2 as both the substrates and solvents. Moreover, only a catalytic amount of $\mathrm{Na}_{2} \mathrm{CO}_{3}$, a very cheap buck chemical, was used as the catalyst in this transformation, which made this chemistry exceptionally appealing for practical application.

Supplementary Materials: Supplementary materials are available online.

Acknowledgments: We are grateful for the financial support from the National Natural Science Foundations of China (No. 81560563, No. 81660576 and No. 81603390); Excellent Creative Talents of Science and Technology Support Plan in Guizhou Province Colleges and Universities ([(2015)491] Qian Jiao He KY Zi); Guizhou Chinese Medicine and Pharmaceutical Engineering Professional Degree Graduate Student Workstation (JYSZ [2014]002); Major Project of the Graduate Student Education Teaching Reform (Qian Jiao Yan He JG Zi)[2016]06; Science and Technology Major Project of Guizhou Province (Qian Ke He J Zhong Da Zi ([2015]6009-5)and Guizhou Education Research Together KYJJ Word ([2016]07).

Author Contributions: B.L. and Z.-Y.C. conceived and designed the experiments; H.-H.L. and Q.-D.W. performed experiments; T.-T.F. and C.W. analyzed data; W.-C.Y. and Y.Z. contributed reagents/materials/analysis tools; X.-L.L. wrote the paper.

Conflicts of Interest: The authors declare no conflict of interest.

\section{References}

1. Marti, C.; Carreira, E.M. Construction of Spiro[pyrrolidine-3,3'-oxindoles]-Recent Applications to the Synthesis of Oxindole Alkaloids. Eur. J. Org. Chem. 2003, 12, 2209-2219. [CrossRef] 
2. Trost, B.M.; Brennan, M.K. Asymmetric Syntheses of Oxindole and Indole Spirocyclic Alkaloid Natural Products. Synthesis 2009, 18, 3003-3025. [CrossRef]

3. Klein, J.E.M.N.; Taylor, R.J.K. Transition-Metal-Mediated Routes to 3,3-Disubstituted Oxindoles through Anilide Cyclisation. Eur. J. Org. Chem. 2011, 34, 6821-6841. [CrossRef]

4. Zhou, F.; Liu, Y.-L.; Zhou, J. Catalytic Asymmetric Synthesis of Oxindoles Bearing a Tetrasubstituted Stereocenter at the C-3 Position. Adv. Synth. Catal. 2010, 352, 1381-1407. [CrossRef]

5. Ball-Jones, N.R.; Badillo, J.J.; Franz, A.K. Strategies for the enantioselective synthesis of spirooxindoles. Org. Biomol. Chem. 2012, 10, 5165-5181. [CrossRef] [PubMed]

6. Dalpozzo, R.; Bartolib, G.; Bencivenni, G. Recent advances in organocatalytic methods for the synthesis of disubstituted 2- and 3-indolinones. Chem. Soc. Rev. 2012, 41, 7247-7290. [CrossRef] [PubMed]

7. Shen, K.; Liu, X.; Lin, L.; Feng, X. Recent progress in enantioselective synthesis of C3-functionalizedoxindoles: Rare earth metals take action. Chem. Sci. 2012, 3, 327-334. [CrossRef]

8. Cheng, D.; Ishihara, Y.; Tan, B.; Barbas, C.F., III. Organocatalytic Asymmetric Assembly Reactions: Synthesis of Spirooxindoles via Organocascade Strategies. ACS Catal. 2014, 4, 743-762. [CrossRef]

9. Galliford, C.V.; Scheidt, K.A. Pyrrolidinyl-Spirooxindole Natural Products as Inspirations for the Development of Potential Therapeutic Agents. Angew. Chem. Int. Ed. 2007, 46, 8748-8758. [CrossRef] [PubMed]

10. Vine, K.L.; Matesic, L.; Locke, J.M.; Ranson, M.; Skropeta, D. Cytotoxic and Anticancer Activities of Isatin and Its Derivatives: A Comprehensive Review from 2000 to 2008. Anti-Cancer Agents Med. Chem. 2009, 9, 397-414. [CrossRef]

11. Badillo, J.J.; Hanhan, N.V.; Franz, A.K. Enantioselective Synthesis of Substituted Oxindoles and Spirooxindoles with Applications in Drug Discovery. Curr. Opin. Drug Discovery Dev. 2010, 13, 758-776.

12. Singh, G.S.; Desta, Z.Y. Isatins as Privileged Molecules in Design and Synthesis of Spiro-Fused Cyclic Frameworks. Chem. Rev. 2012, 112, 6104-6155. [CrossRef] [PubMed]

13. Hong, L.; Wang, R. Recent Advances in Asymmetric Organocatalytic Construction of 3,3'-Spirocyclic Oxindoles. Adv. Synth. Catal. 2013, 355, 1023-1052. [CrossRef]

14. Hinman, R.L.; Bauman, C.P. Reactions of 3-Bromooxindoles. The Synthesis of 3-Methyleneoxindole. J. Org. Chem. 1964, 29, 2431-2437. [CrossRef]

15. Fuchs, J.R.; Funk, R.L. Indol-2-one Intermediates: Mechanistic Evidence and Synthetic Utility. Total Syntheses of ( \pm )-Flustramines A and C. Org. Lett. 2005, 7, 677-680. [CrossRef] [PubMed]

16. Fuchs, J.R.; Funk, R.L. Total Synthesis of ( \pm )-Perophoramidine. J. Am. Chem. Soc. 2004, 126, 5068-5069. [CrossRef] [PubMed]

17. Zuo, J.; Liao, Y.-H.; Zhang, X.; Yuan, W.-C. Organocatalyzed Enantioselective Decarboxylative Stereoablation Reaction for the Construction of 3,3'-Disubstituted Oxindoles Using $\beta$-Ketoacids and 3-Halooxindoles. J. Org. Chem. 2012, 77, 11325-11332. [CrossRef] [PubMed]

18. Liao, Y.-H.; Wu, Z.-J.; Han, W.-Y.; Zhang, X.-M.; Yuan, W.-C. Organocatalytic Enantioselective Stereoablative Hydroxylation of 3-Halooxindoles: An Effective Method for the Construction of Enantioenriched 3-Substituted 3-Hydroxy-2-Oxindoles. Chem. Eur. J. 2012, 18, 8916-8920. [CrossRef] [PubMed]

19. Ma, S.; Han, X.; Krishnan, S.; Virgil, S.C.; Stoltz, B.M. Catalytic Enantio selective Stereoablative Alkylation of 3-Halooxindoles: Facile Access to Oxindoles with C3 All-Carbon Quaternary Stereocenters. Angew. Chem. Int. Ed. 2009, 48, 8037-8041. [CrossRef] [PubMed]

20. Zhang, H.; Hong, L.; Kang, H.; Wang, R. Construction of Vicinal All-Carbon Quaternary Stereocenters by Catalytic Asymmetric Alkylation Reaction of 3-Bromooxindoles with 3-Substituted Indoles: Total Synthesis of (+)-Perophoramidine. J. Am. Chem. Soc. 2013, 135, 14098-14101. [CrossRef] [PubMed]

21. Zuo, J.; Wu, Z.-J.; Zhao, J.-Q.; Zhou, M.-Q.; Xu, X.-Y.; Zhang, X.-M.; Yuan, W.-C. Preparation of 3-Sulfonylated 3,3-Disubstituted Oxindoles by the Addition of Sulfinate Salts to 3-Halooxindoles. J. Org. Chem. 2015, 80, 634-640. [CrossRef] [PubMed]

22. Krishnan, S.; Stoltz, B.M. Preparation of 3-Sulfonylated 3,3-Disubstituted Oxindoles by the Addition of Sulfinate Salts to 3-Halooxindoles. Tetrahedron Lett. 2007, 48, 7571-7573. [CrossRef]

23. Zhao, M.; Li, N.-K.; Zhang, Y.-F.; Pan, F.-F.; Wang, X.-W. Construction of 3-amino-2-oxindoles by direct amination of aniline or $\alpha$-amino-acid derivatives to 3-bromooxindoles. Tetrahedron 2016, 72, 1406-1414. [CrossRef] 
24. Zhang, H.; Kang, H.; Hong, L.; Dong, W.; Li, G.; Zheng, X.; Wang, R. Construction of the N1-C3 Linkage Stereogenic Centers by Catalytic Asymmetric Amination Reaction of 3-Bromooxindoles with Indolines. Org. Lett. 2014, 16, 2394-2397. [CrossRef] [PubMed]

25. Wu, C.; Li, G.; Sun, W.; Zhang, M.; Hong, L.; Wang, R. Organocatalytic Highly Enantioselective Monofluoroalkylation of 3-Bromooxindoles: Construction of Fluorinated 3,3'-Disubstituted Oxindoles and Their Derivatives. Org. Lett. 2014, 16, 1960-1963. [CrossRef] [PubMed]

26. Bai, X.; Jing, Z.; Liu, Q.; Ye, X.; Zhang, G.; Zhao, X.; Jiang, Z. L-Amino Acid Based Urea-Tertiary Amine-Catalyzed Chemoselective and Asymmetric Stereoablative Carboxylation of 3-Bromooxindoles with Malonic Acid Half Thioesters. J. Org. Chem. 2015, 80, 12686-12696. [CrossRef] [PubMed]

27. Frederick, W.; Goldberg, P.M.; Rachel, T. A Mild Thermal and Acid-Catalyzed Rearrangement of O-Aryl Ethers intoortho-HydroxyArenes. Org. Lett. 2005, 7, 4531-4534.

28. Dyachenko, V.I.; Peregudov, A.S.; Chkanikov, N.D. Trifluoromethyl-containing N-acylmethylenequinone imines as novel highly electrophilic agents. J. Fluor. Chem. 2007, 128, 868-878. [CrossRef]

29. Wang, Y.; Tang, X.; Shao, Z.; Ren, J. Indole-based alkaloids from deep-sea bacterium Shewanellapiezotolerans with antitumor activities. J. Antibiot. 2014, 67, 395-399. [CrossRef] [PubMed]

30. Subba Reddy, B.-V.; Rajeswari, N.; Sarangapani, M.; Prashanthi, Y.; Ganji, R.-J.; Addlagatta, A. Iodine-catalyzed condensation of isatin with indoles: A facile synthesis of di(indolyl)indolin-2-ones and evaluation of their cytotoxicity. Bioorg. Med. Chem. Lett. 2012, 22, 2460-2463. [CrossRef] [PubMed]

31. Kamal, A.; Srikanth, Y.-V.-V.; Khan, M.-N.-A.; Shaik, T.-B.; Ashraf, M. Synthesis of 3,3-diindolyl oxyindoles efficiently catalysed by $\mathrm{FeCl} 3$ and their in vitro evaluation for anticancer activity. Bioorg. Med. Chem. Lett. 2010, 20, 5229-5231. [CrossRef] [PubMed]

32. Paira, P.; Hazra, A.; Kumar, S.; Paira, R.; Sahu, K.-B.; Naskar, S.; Saha, P.; Mondal, S.; Maity, A.; Banerjee, S.; et al. Efficient synthesis of 3,3-diheteroaromatic oxindole analogues and their in vitro evaluation for spermicidal potential. Bioorg. Med. Chem. Lett. 2009, 19, 4786-4789. [CrossRef] [PubMed]

33. Hibino, S.; Choshi, T. Simple indole alkaloids and those with a nonrearranged monoterpenoid unit. Nat. Prod. Rep. 2001, 18, 66-87. [CrossRef] [PubMed]

34. Marsden, S.P.; Watson, E.L.; Raw, S.A. Facile and General Synthesis of Quaternary 3-Aminooxindoles. Org. Lett. 2008, 10, 2905-2908. [CrossRef] [PubMed]

35. Snell, R.-H.; Woodward, R.-L.; Willis, M.-C. Catalytic Enantio selective Total Synthesis of Hodgkinsine B. Angew. Chem. Int. Ed. 2011, 50, 9116-9119. [CrossRef] [PubMed]

36. Boyer, N.; Movassaghi, M. Concise total synthesis of (+)-gliocladins B and C. Chem. Sci. 2012, 3, $1798-1803$. [CrossRef] [PubMed]

37. Luo, L.; Zhang, J.-J.; Ling, W.-J.; Shao, Y.-L.; Wang, Y.-W.; Peng, Y. Unified Synthesis of (-)-Folicanthine and (-)-Ditryptophenaline Enabled by a Room Temperature Nickel-Mediated Reductive Dimerization. Synthesis 2014, 46, 1908-1915. [CrossRef]

38. Li, T.-Z.; Wang, X.-B.; Sha, F.; Wu, X.-Y. Catalytic enantioselective addition of alcohols to isatin-derived N-Bocketimines. Tetrahedron 2013, 69, 7314-7319. [CrossRef]

39. Jhan, Y.-H.; Kang, T.-W.; Hsieh, J.-C. Efficient copper-catalyzed intramolecular N-arylation for the synthesis of oxindoles. Tetrahedron Lett. 2013, 54, 1155-1159.

40. Hsieh, J.-C.; Cheng, A.-Y.; Fu, J.-H.; Kang, T.-W. Copper-catalyzed domino coupling reaction: An efficient method to synthesize oxindoles. Org. Biomol. Chem. 2012, 10, 6404-6409. [CrossRef] [PubMed]

41. Piemontesi, C.; Wang, Q.; Zhu, J. Synthesis of 3,3-disubstituted oxindoles by one-pot integrated Brønsted base-catalyzed trichloroacetimidation of 3-hydroxyoxindoles and Brønsted acid-catalyzed nucleophilic substitution reaction. Org. Biomol. Chem. 2013, 11, 1533-1536. [CrossRef] [PubMed]

Sample Availability: Samples of the compounds 3aa-3ah are available from the authors. 